\title{
Analytic Novikov for topologists
}

\section{Jonathan Rosenberg}

Abstract. We explain for topologists the "dictionary" for understanding the analytic proofs of the Novikov conjecture, and how they relate to the surgery-theoretic proofs. In particular, we try to explain the following points:

(1) Why do the analytic proofs of the Novikov conjecture require the introduction of $C^{*}$-algebras?

(2) Why do the analytic proofs of the Novikov conjecture all use $K$ theory instead of $L$-theory? Aren't they computing the wrong thing?

(3) How can one show that the index map $\mu$ or $\beta$ studied by operator theorists matches up with the assembly map in surgery theory?

(4) Where does "bounded surgery theory" appear in the analytic proofs? Can one find a correspondence between the sorts of arguments used by analysts and the controlled surgery arguments used by topologists?

The literature on the Novikov conjecture (see $[\mathrm{FRR}]$ ) consists of several different kinds of papers. Most of these fall into two classes: those based on topological arguments, usually involving surgery theory, and those based on analytic arguments, usually involving index theory. The purpose of this note is to "explain" the second class of papers to those familiar with the first class. I do not intend here to give a detailed sketch of the Kasparov $K K$-approach to the Novikov conjecture (for which the key details appear in [Kas4], [Fac2], and [KS]), since this has already been done in the convenient expository references [Fac1], [Kas2], [Kas3], [Bla], and [Kas5]. Nor do I intend to explain the approach to the Novikov conjecture taken by Mishchenko and Soloviev (found in [Mis1], [Mis2], [MS], [Mis3], and [KS, Appendix]), using Fredholm representations but not using $K K$, for which a convenient expository reference is $[\mathrm{HsR}]$. Rather, I intend to concentrate on explaining the "dictionary" for relating the two main classes of papers on the Novikov conjecture, the topological and the analytic, and on trying

1980 Mathematics Subject Classification (1985 Revision). Primary 19J25; Secondary 46L80, 19G24, 19K35, 19K56, 57R67, 55P42, 58G12.

Expanded version of a lecture given at the Oberwolfach meeting on "Novikov conjectures, index theorems and rigidity," 6 September, 1993. I would like to thank my co-organizers Steve Ferry and Andrew Ranicki for encouraging me to write this talk up for publication, and also for useful technical comments. I would also like to thank Erik Pedersen, John Roe, Stephan Stolz, Shmuel Weinberger, and the referee for helpful suggestions about the exposition. Finally, I would like to thank Bruce Williams for noticing a homotopy-theoretic error in the original version of $\S 2$.

This work was partially supported by NSF Grant \# DMS-92-25063. 
to find the common ground relating them. I will assume that the reader is already familiar with the reduction of Novikov's original conjecture on homotopy invariance of higher signatures to a statement about the $L$-theory assembly map

$$
A: H_{\bullet}\left(X ; \mathbb{L}_{\bullet}(\mathbb{Z})\right) \rightarrow L_{\bullet}\left(\mathbb{Z} \pi_{1}(X)\right),
$$

in the case where the space $X$ is taken to be a $K(\pi, 1)$-space $B \pi$. This aspect of the problem is discussed elsewhere in these proceedings, especially in $[\operatorname{Ran} 3]$.

\section{$\S 1$. Why $K$-theory of $C^{*}$-algebras?}

Topologists looking at the analytic literature on the Novikov conjecture often wonder why so much emphasis is placed on the (topological) $K$-theory of $C^{*}$-algebras, when in fact it is known that the original Novikov conjecture has to do with $L$-theory of group rings, and that certain related problems in the topology of non-simply connected manifolds (concerning Whitehead torsion) have to do with the algebraic $K$-theory of integral group rings. First let's pin down the objects of study.

1.1. Definition. A Banach *-algebra is a real or complex Banach algebra, together with an isometric (conjugate-linear) involution *. A *-homomorphism or $*$-isomorphism of such algebras means a homomorphism or isomorphism preserving the involutions. A real or complex $C^{*}$-algebra $A$ is a Banach $*$-algebra which is isometrically $*$-isomorphic to a norm-closed involutive subalgebra of the bounded operators on some Hilbert space (real or complex, as the case may), with involution obtained by sending an operator $a$ to its Hilbert space adjoint $a^{*}$, defined by the property that

$$
\langle a \xi, \eta\rangle=\left\langle\xi, a^{*} \eta\right\rangle
$$

(In the real case, it is important to remember that real, complex, and quaternionic Hilbert spaces may all be regarded by restriction of scalars as real Hilbert spaces.) If a $C^{*}$-algebra $A$ acts on a Hilbert space $\mathcal{H}$, then $M_{n}(A)$ naturally acts on the Hilbert space $\mathcal{H}^{n}$, and thus any matrix algebra over a $C^{*}$-algebra is also a $C^{*}$-algebra.

We also quickly remind the reader of the most crucial special properties of $C^{*}$-algebras, which may be deduced either from the Spectral Theorem for self-adjoint operators on a Hilbert space, or else from the algebraic characterization of $C^{*}$-algebras as in [Ped, Ch. 1]. First, some basic definitions.

1.2. Definition. An element $a$ of a Banach *-algebra $A$ is called selfadjoint if $a=a^{*}$, positive (actually "non-negative" would be better, since 
0 is not excluded, but the name "positive" has become standard), written $a \geq 0$, if $a=b^{*} b$ for some other element $b$. If $A$ has a unit and $a \in A$, the spectrum spec $a$ of $a$ (this has nothing to do with spectra in homotopy theory!!) is the set of $\lambda \in \mathbb{C}$ such that $a-\lambda \cdot 1$ is not invertible in $A$ (or in $A_{\mathbb{C}}:=A \otimes_{\mathbb{R}} \mathbb{C}$ if $A$ is only an algebra over $\mathbb{R}$ ). This is always a compact subset of $\mathbb{C}$, and clearly it only depends on the structure of $A$ as an algebra over $\mathbb{C}$ or $\mathbb{R}$, not on the norm. Note that in the real case, since the obvious action of $\operatorname{Gal}(\mathbb{C} / \mathbb{R})$ on $A_{\mathbb{C}}$ fixes $a \in A$, it follows that $\operatorname{spec} a$ is invariant under complex conjugation. If $A$ does not have a unit, then we may always embed $A$ as an ideal of codimension 1 in a $C^{*}$-algebra $A_{+}$with unit, obtained by realizing $A$ as an algebra of operators on a Hilbert space $\mathcal{H}$ and considering all operators of the form $a+\lambda \cdot 1$, where $\lambda$ is a scalar and 1 is the identity operator on $\mathcal{H}$. The spectrum of $a \in A$ may then be defined to be its spectrum in $A_{+}$, and in this case, spec $a$ always contains 0 .

Here are the crucial facts we will need.

1.3. Facts. Let $A$ be a real or complex $C^{*}$-algebra, and let $a \in A$.

(i) If $a$ is self-adjoint, then spec $a \subset \mathbb{R}$. Conversely, if $a$ and $a^{*}$ commute and spec $a \subset \mathbb{R}$, then $a=a^{*}$.

(ii) If $a \geq 0$, then $\operatorname{spec} a \subset[0, \infty)$. Conversely, if $a=a^{*}$ and $\operatorname{spec} a \subset$ $[0, \infty)$, then $a \geq 0$.

(iii) (Functional Calculus) Assume that $a=a^{*}$, or more generally that $a$ and $a^{*}$ commute, and that $f$ is a continuous function defined on spec $a$. (If $A$ is an algebra over $\mathbb{C}, f$ can be complex-valued; if $A$ is an algebra over $\mathbb{R}$, then $f$ has to satisfy the condition that $\overline{f(z)}=f(\bar{z})$.) Assume in addition that if $A$ does not have a unit, then $f(0)=0$. Then there is a unique element $f(a) \in A$ which is contained in the closed subalgebra generated by $a$, having the property that if $f_{n}$ is a sequence of polynomials (chosen without constant term, in case $A$ does not have a unit), converging uniformly to $f$ on $\operatorname{spec} a$, then $f_{n}(a)$ (defined as in any algebra) converges in norm to $f(a)$.

(iv) Any *-homomorphism $\varphi$ from $A$ to another $C^{*}$-algebra is automatically continuous with closed range, and induces an isometric *isomorphism from $A / \operatorname{ker} \varphi$ onto range $\varphi$.

(v) If $A$ has a unit, any finitely generated projective $A$-module defined by an idempotent $e=e^{2}$ in $A$ may also be defined by a self-adjoint idempotent $p$ in $A$.

(vi) The norm on $A$ is determined by the *-algebra structure by the formula $\|a\|^{2}=\max \operatorname{spec} a^{*} a$.

Proof. We omit the proofs of (i)-(iv), which can be found in [Ped, Ch. 1], except for the real case of (iii). Here one first uses the complex case to define 
$f(a)$ as an element of $A_{\mathbb{C}}$, and then one uses the condition $\overline{f(z)}=f(\bar{z})$ to see that $f(a)$ is invariant under the action of $\operatorname{Gal}(\mathbb{C} / \mathbb{R})$ and thus lies in $A$. The property (vi) follows from basic facts about operators on Hilbert spaces.

Since (v) is a little less standard (I believe it was first noted by Kaplansky), we include a proof. One can do everything completely algebraically, but to get a better impression of what is going on, suppose $A$ is acting on a Hilbert space $\mathcal{H}$. Then the image of $e$ must be a closed subspace $V$ of $\mathcal{H}$, and with respect to the decomposition $\mathcal{H}=V \oplus V^{\perp}$ of $\mathcal{H}$, e must have a matrix of the form $\left(\begin{array}{ll}1 & a \\ 0 & 0\end{array}\right)$, where $a: V^{\perp} \rightarrow V$ is a bounded operator. Then

$$
e^{*}=\left(\begin{array}{cc}
1 & 0 \\
a^{*} & 0
\end{array}\right), \quad e e^{*}=\left(\begin{array}{cc}
1+a a^{*} & 0 \\
0 & 0
\end{array}\right),
$$

and in particular, the spectrum of $e e^{*}$ is contained in $\{0\} \cup[1, \infty)$. Thus if $f(0)=0$ and $f(t)=1$ for $t \geq 1, f$ is continuous on the spectrum of $e e^{*}$ and thus (by (iii) above) $p=f\left(e e^{*}\right)$ lies in $A$ and is a self-adjoint projection with the same range as $e$. We claim $A e \cong A p$ as projective modules over $A$. But in fact $e p=p$ and $p e=e$, so $A e$ and $A p$ are not only isomorphic but equal (as subsets of $A$ ).

We should emphasize that these facts are special to $C^{*}$-algebras; they fail in some other Banach $*$-algebras.

1.4. To explain the appearance of $K$-theory of group $C^{*}$-algebras, consider a group $\pi$ with integral group ring $\mathbb{Z} \pi$ and complex group ring $\mathbb{C} \pi$. One can form the (complex) Hilbert space $\ell^{2}(\pi)$ having $\pi$ as an orthonormal basis, and by definition, the reduced group $C^{*}$-algebra $C_{r}^{*}(\pi)$ is the completion of $\mathbb{C} \pi$ in the operator norm for its action on $\ell^{2}(\pi)$ by left multiplication. Most analytic approaches to the Novikov conjecture for the group $\pi$, and thus to homotopy invariance of higher signatures for manifolds having $\pi$ as fundamental group, involve in some way the $K$-theory of the $C^{*}$-algebra $C_{r}^{*}(\pi)$. While there are obvious inclusions

$$
\mathbb{Z} \pi \hookrightarrow \mathbb{C} \pi \hookrightarrow C_{r}^{*}(\pi)
$$

it is not in any way expected that these should be close to inducing isomorphisms on $K$-theory. In fact, in the fundamental example where $\pi \cong \mathbb{Z}^{n}$ is free abelian, $\tilde{K}_{0}$ vanishes for both $\mathbb{Z} \pi$ and $\mathbb{C} \pi$, whereas $K_{0}\left(C_{r}^{*}(\pi)\right) \cong$ $K^{0}\left(T^{n}\right)$ has rank $2^{n-1}$. Rather, the introduction of $K_{0}\left(C_{r}^{*}(\pi)\right)$ is designed to facilitate the definition of a "non-simply connected Hirzebruch signature formula," which Novikov recognized from the start to be intimately tied up with the conjecture on higher signatures. In fact, an examination 
of Novikov's original text in [Nov, $§ 11]$ shows that he already suggested the introduction of $C^{*}$-algebras for this purpose, though only in a special case. (For the original text and an analysis of Novikov's formulation of the higher signature conjecture, see [FRR, §2].)

1.5. To see how $C^{*}$-algebras come in, it is worth thinking about how the ordinary signature of a manifold is defined. The Poincaré duality structure (which is the homotopy-theoretic manifestation of the basic geometric property of transversality) gives rise to a symmetric bilinear form on middledegree cohomology. The signature is extracted from this form by diagonalizing the form (over $\mathbb{R}$ ) and taking the formal difference of its positive and negative eigenspaces. Thus while it is customary to view the signature as an ordinary integer, it naturally arises as an element of a group of such formal differences, that is, as an element of $K_{0}(\mathbb{R})$ (which happens to be naturally isomorphic to $\mathbb{Z}$ ). It is thus natural to expect a "generalized signature" also to be a formal difference of positive and negative eigenspaces of a bilinear form, this time arising from Poincaré duality with local coefficients. For this to make sense, it is necessary to work in a ring over which the form is diagonalizable, and this is where $C^{*}$-algebras come in. To "diagonalize" a form, one wants to make use of the Spectral Theorem for self-adjoint operators on a Hilbert space, but this requires that our involutive ring be identifiable with a ring of bounded operators on a Hilbert space, in other words, with a (subalgebra of a) $C^{*}$-algebra. Then the "positive and negative eigenspaces" of the form will be projective modules over this ring, and thus the signature, their formal difference, will be an element of $K_{0}$ of a $C^{*}$-algebra completion of the (real or complex) group ring. Signatures are more tractable objects than forms, since (topological) $K$-theory behaves better than $L$-theory, because of its useful "rigidity" properties of homotopy invariance and strong excision. Thus the whole machinery of topological $K$-theory and index theory can be brought to bear on the study of "generalized signatures."

An important question in trying to prove the Novikov conjecture using analytic methods is whether the passage from symmetric forms to signatures loses any essential information. One may view this operation as consisting of two rather different steps: passage from symmetric forms over the group ring to symmetric forms over a completed group ring, and passage from symmetric forms to signatures in the context of $C^{*}$-algebras. The first of these operations is still not completely understood, though it seems that in many cases it loses information only at the prime 2. (As an example, the natural maps $L_{\bullet}(\mathbb{Z}) \rightarrow L_{\bullet}(\mathbb{R})$ are not isomorphisms in even degrees, but become isomorphisms after tensoring with $\mathbb{Z}\left[\frac{1}{2}\right]$.) However, the second operation, passing from forms to signatures in the context of $C^{*}$-algebras, is better understood, and loses almost no information. A key folk theorem 
in this direction, which I believe may have first been discovered by Gelfand or Mishchenko, is often cited but hard to pin down in the literature, ${ }^{*}$ so we include a proof here. The case where $A=C^{\mathbb{C}}(X)$ is complex and abelian appears in $[\mathrm{GM}]$ and as Theorem 4.1 in [Nov].

1.6. Theorem (Folklore). Let $A$ be a real or complex $C^{*}$-algebra with unit, regarded also as ring with involution (in the complex case, note that the involution on scalar multiples of the identity is complex conjugation). If $\varphi$ is a non-singular hermitian form on a finitely generated projective $A$ module $P$, then there is a unique $\varphi$-invariant splitting $P=P^{+} \oplus P^{-}$of $P$, with respect to which $\varphi$ is equivalent to the form with matrix

$$
\left(\begin{array}{cc}
1_{P^{+}} & 0 \\
0 & -1_{P^{-}}
\end{array}\right) .
$$

Thus isomorphism classes of non-singular hermitian forms over $A$ may be identified with pairs $\left(\left[\mathrm{P}^{+}\right],\left[\mathrm{P}^{-}\right]\right)$of isomorphism classes of finitely generated projective modules over $A$. The "signature" map

$$
[P, \varphi] \mapsto\left[P^{+}\right]-\left[P^{-}\right]
$$

defines a natural isomorphism of functors

$$
\Phi: L_{0} \stackrel{\cong}{\longrightarrow} K_{0}
$$

(on the category of real or complex $C^{*}$-algebras with unit), where $L_{0}=L_{0}^{p}$ is the usual projective quadratic L-group of $A$ as defined in [Ran1, §1] (this coincides with the symmetric $L$-group $L^{0}$ since $\frac{1}{2} \in A$ ).

Similarly, if $\varphi$ is a non-singular skew-hermitian form on a finitely generated free $A$-module $P=A^{n}$, then $\varphi$ is equivalent to the form defined by a skew-hermitian element $h$ of $M_{n}(A)$ with spec $h \subset\{i,-i\}$. (If $A$ is an algebra over $\mathbb{C}$, then since $i=\sqrt{-1} \in A$,

$$
(x, y) \mapsto \varphi(i x, y)
$$

is a non-singular hermitian form, so in the complex case there is no essential difference between hermitian and skew-hermitian forms.)

Proof. Recall that $L^{0}(A)=L_{0}(A)$ is the Witt group of non-singular hermitian forms $\varphi$ defined on finitely generated projective (left) $A$-modules $P$,

*Equivalent statements using slightly different notation appear in [Mis2], [Kar3] and in $[\mathrm{KM}]$. 
modulo hyperbolic forms. Let $(P, \varphi)$ be such a form, and let $Q$ be a complement to $P$, so that $P \oplus Q$ is finitely generated and free, say $P \oplus Q \cong A^{n}$. We may identify $P$ with $A^{n} e$, where $e$ is a self-adjoint projection in $M_{n}(A)$ (which is 0 on $Q$ and the identity on $P$ ), and may identify $\varphi$ with an element $h=h^{*} \in e M_{n}(A) e$. (We have used Fact 1.3(v).) Non-singularity of $\varphi$ means that $h+1-e$ is invertible, and thus (using Fact 1.3(i)) its spectrum is contained in

$$
(\infty,-\varepsilon] \cup[\varepsilon, \infty)
$$

for some $\varepsilon>0$ (depending on $h$ ). Let $f$ be +1 on $[\varepsilon, \infty)$ and 0 on $(\infty,-\varepsilon]$. Then (using Fact 1.3(iii)) $f(h+1-e)$ is a self-adjoint projection which commutes with $h$ and $e$; also it is clear that $f(h+1-e) \geq 1-e$ (in other words, if $p=f(h+1-e)-(1-e)$, then $0 \leq p \leq e)$. Note that $P=P p \oplus P(1-p)=P^{+} \oplus P^{-}$is exactly the decomposition of $P$ into the "positive" and "negative" eigenspaces of $\varphi$, as in the definition of the classical signature in 1.5 , in that $h p \geq 0$ and $h(1-p) \leq 0$. It is obvious from the construction that the submodules $P^{+}$and $P^{-}$are $\varphi$-invariant. So to show that $\varphi$ is equivalent to the form with matrix

$$
\left(\begin{array}{cc}
1_{P^{+}} & 0 \\
0 & -1_{P^{-}}
\end{array}\right)
$$

it is sufficient to replace $P$ by $P^{+} \oplus$ (some complement) and $h$ by $h p \oplus 1$, and to show show that if the form $\varphi$ on a finitely generated free module $A^{n}$ is given by an invertible positive operator $h$, then it is equivalent to the standard form

$$
\langle a, b\rangle=a_{1} b_{1}^{*}+\cdots+a_{n} b_{n}^{*} \cdot \dagger
$$

(A similar argument then applies to $P^{-}$.)

But the fact that $\varphi$ is given by $h$ means of course that

$$
\varphi(a, b)=a h b^{*}
$$

Since $h$ is positive, it has a square root $h^{\frac{1}{2}}$ (defined using Fact 1.3(iii)), and $h^{\frac{1}{2}}$ is invertible since $h$ is. Thus under the change of basis defined by $h^{-\frac{1}{2}}, h$ is replaced by $h^{-\frac{1}{2}} h h^{-\frac{1}{2}}{ }^{*}=1$, as required. On the other hand, any change of basis sends $h$ to $k h k^{*}$ for some $k$, and thus preserves positivity. So the splitting into positive and negative eigenspaces is unique, and this proves the part of the theorem about classification of forms.

$\dagger \mathrm{I}$ am taking forms to be $A$-linear in the first variable and $*$-linear in the second variable, slightly different notation from that used in [Ran1]. 
To complete the proof in the hermitian case, observe that we now know that non-singular hermitian forms over $A$ correspond exactly to pairs $\left(P^{+}\right.$, $P^{-}$) of finitely generated projective modules. Hyperbolic forms obviously correspond to such pairs with $P^{+} \cong P^{-}$. So the "signature" $\left[P^{+}\right]-\left[P^{-}\right]$ is well-defined on Witt classes and clearly induces an isomorphism from $L^{0}=L_{0}$ to $K_{0}$.

As far as the skew-hermitian case is concerned, basically the same proof shows that if $P=A^{n} e$, where $e$ is a self-adjoint projection in $M_{n}(A)$, then using the functional calculus we can change variables so that $\varphi$ is given by (the cut-down to $P$ of) a skew-hermitian element $h$ of $M_{n}(A)$ with spec $h \subset\{i,-i\}$ and commuting with the projection $e$. In the complex case, $h$ is diagonalizable and the classification is the same as in the hermitian case. In the real case, this is not quite enough to classify the skew-hermitian forms.

1.7. Remark. The functor $L_{0}^{h}$ defined using hermitian forms on finitely generated free modules does not have such a simple description, but one can see from the above description that for $A$ as above, $L_{0}^{h}(A)$ can be identified with the quotient group

$$
\begin{array}{r}
\left\{\left(\left[P^{+}\right],\left[P^{-}\right]\right) \in K_{0}(A) \times K_{0}(A):\left[P^{+}\right]+\left[P^{-}\right]=0 \text { in } \tilde{K}_{0}(A)\right\} \\
/\langle([A],[A])\rangle .
\end{array}
$$

This is consistent with the usual Rothenberg exact sequence (derived for example in [Ran1, §1.10])

$$
\cdots \rightarrow \hat{H}^{1}\left(\mathbb{Z} / 2 ; \tilde{K}_{0}(A)\right) \rightarrow L_{0}^{h}(A) \rightarrow L_{0}^{p}(A) \rightarrow \hat{H}^{0}\left(\mathbb{Z} / 2 ; \tilde{K}_{0}(A)\right) \rightarrow \cdots
$$

since we see that when $L_{0}^{p}(A)$ is identified with $K_{0}(A)$, the image of $L_{0}^{h}(A)$ consists of all

$$
\left[P^{+}\right]-\left[P^{-}\right], \quad\left[P^{+}\right]+\left[P^{-}\right]=0 \text { in } \tilde{K}_{0}(A)
$$

and thus the cokernel of $L_{0}^{h}(A) \rightarrow L_{0}^{p}(A)$ is just

$$
\tilde{K}_{0}(A) / 2 \tilde{K}_{0}(A)
$$

Note that this is $\hat{H}^{0}\left(\mathbb{Z} / 2 ; \tilde{K}_{0}(A)\right)$ for the trivial action of $\mathbb{Z} / 2$ on $\tilde{K}_{0}(A)$. However, the trivial action is indeed appropriate here, since the action of $\mathbb{Z} / 2$ on $\tilde{K}_{0}(A)$ in the Rothenberg sequence is defined via the map $p \mapsto p^{*}$ on idempotents. But every finitely generated projective module over a $C^{*}$ algebra may be defined by a self-adjoint idempotent in some matrix algebra over $A$, because of Fact $1.3(\mathrm{v})$. 
Similarly, the kernel of $L_{0}^{h}(A) \rightarrow L_{0}^{p}(A)$ is given by

$$
\begin{array}{r}
\left\{([P],[P]):[P] \in K_{0}(A), \quad 2[P]=0 \quad \text { in } \tilde{K}_{0}(A)\right\} /\langle([A],[A])\rangle \\
\cong 2 \text {-torsion in } \tilde{K}_{0}(A) \cong \hat{H}^{1}\left(\mathbb{Z} / 2 ; \tilde{K}_{0}(A)\right) .
\end{array}
$$

Thus for $C^{*}$-algebras the Rothenberg sequence reduces to

$$
0 \rightarrow \hat{H}^{1}\left(\mathbb{Z} / 2 ; \tilde{K}_{0}(A)\right) \rightarrow L_{0}^{h}(A) \rightarrow L_{0}^{p}(A) \rightarrow \hat{H}^{0}\left(\mathbb{Z} / 2 ; \tilde{K}_{0}(A)\right) \rightarrow 0,
$$

with $\mathbb{Z} / 2$ acting trivially.

While Theorem 1.6 seems to be well-known, the relationship between higher $L$-groups and higher $K$-groups for $C^{*}$-algebras is more complicated and does not seem to be explained very clearly in the literature. Recall that for a Banach algebra $A$, possibly without unit, one has topological $K$ groups $K_{\bullet}^{\text {top }}(A)$ (which for $\bullet \geq 1$ are just the homotopy groups of $G L(A)$ shifted in degree) which are periodic of period 2 in the complex case and of period 8 in the real case (convenient references are [Kar2] and [Bla]). For algebras of functions, these are the more familiar groups (to topologistsoperator algebraists use the groups $K_{\bullet}^{\text {top }}(A)$ all the time!) $K_{\bullet}^{\text {top }}\left(C^{\mathbb{C}}(X)\right) \cong$ $K U^{-\bullet}(X), K_{\bullet}^{\text {top }}\left(C^{\mathbb{R}}(X)\right) \cong K O^{-\bullet}(X)$. If $(X, \tau)$ is a compact Real space in the sense of Atiyah (in other words, a space with an involution $\tau$ ) and we define

$$
C(X, \tau):=\left\{f \in C^{\mathbb{C}}(X): \overline{f(x)}=f(\tau(x))\right\},
$$

then $K_{\bullet}^{\text {top }}(C(X, \tau)) \cong K R^{-\bullet}(X, \tau) . K_{0}^{\text {top }}$ coincides with the algebraic $K$ group $K_{0}$, but in general, $K_{n}^{\text {top }}$ differs from the algebraic $K$-group $K_{n}$ if $n \neq 0$. On the other hand, we also have the surgery groups $L_{\bullet}^{h}(A)$ and $L_{\bullet}^{p}(A)$ defined forgetting the topology of $A$ and only using the structure of $A$ as a ring with involution. For complex $C^{*}$-algebras $A$, we will see now that the groups $L_{\bullet}^{p}(A)$ and $K_{\bullet}^{\text {top }}(A)$ are naturally isomorphic, whereas for real $C^{*}$-algebras, this is only true after inverting 2 . The coincidence of $L_{\bullet}^{p}(A)$ with $K_{\bullet}^{\text {top }}(A)$ was first noted for commutative complex $C^{*}$-algebras in [Nov, Theorem 4.1], and (for general complex $C^{*}$-algebras) is basically equivalent to some of the results in $\S 3$ and $\S 4$ of [Mis2] (though it's hard at first to see whether that paper is talking about $L_{\bullet}^{h}$ or $L_{\bullet}^{p}$ ). A complete proof, using algebraic surgery and rather different from the one that we will give below, was given in [Mil]. That algebraic L-theory can recapture topological $K$-theory seems surprising until one remembers Fact 1.3(vi), which asserts that the structure of a $C^{*}$-algebra as a topological algebra can be recaptured from its algebraic structure as an involutive algebra over $\mathbb{R}$ or $\mathbb{C}$ (together with the topology of the real or complex numbers). 
1.8. Theorem ([Mis2], [Mil]). Let $A$ be a complex $C^{*}$-algebra with unit. Let $L_{\bullet}(A)$ denote the 4-periodic (projective) quadratic $L$-groups of $A$ as defined in [Ran1, §1] (these also coincide with the symmetric L-groups since $\left.\frac{1}{2} \in A\right)$. Then these groups are 2-periodic, and are naturally isomorphic to the topological $K$-groups $K_{\bullet}^{\text {top }}(A)$.

Proof. The fact that the $L$-groups are 2-periodic is due to the fact that $\sqrt{-1} \in A$, so that there is a natural bijection between hermitian and skewhermitian forms over $A$. Because of Theorem 1.6 and Remark 1.7, we really only need to show that there is a natural isomorphism $\Phi$ from $L_{1}^{h}(A)$ to $K_{1}^{\text {top }}(A)$, analogous to the signature map of Theorem 1.6.

Now the group $L_{1}^{h}(A)$ is defined** to be the limit (as $n \rightarrow \infty$ ) of the abelianization of what we can call $U(n, n ; A)$, the unitary group of the hyperbolic form on $A^{n} \oplus A^{n}$ defined by the matrix

$$
\sigma_{n}=\left(\begin{array}{cc}
0 & 1_{n} \\
1_{n} & 0
\end{array}\right)
$$

modulo $\sigma_{n}$ itself and modulo the image of $G L(n, A)$ under the map

$$
G L(n, A) \rightarrow U(n, n ; A): a \mapsto\left(\begin{array}{cc}
a & 0 \\
0 & \left(a^{*}\right)^{-1}
\end{array}\right)
$$

In the notation of [Kar3], the abelianization of $U^{\text {hyp }}(A):=\underline{\lim } U(n, n ; A)$ is denoted ${ }_{1} L_{1}(A)$. The map $G L(n, A) \rightarrow U(n, n ; A)$ defines in the limit (after passing to abelianizations) a map $K_{1}(A) \rightarrow{ }_{1} L_{1}(A)$, whose cokernel in [Kar3] is denoted ${ }_{1} W_{1}(A)$. However, $G L(n, A)$ and $U(n, n ; A)$ are topological groups (in fact, Banach Lie groups), and if we divide by the connected components of the identity, $G L(n, A)_{0}$ and $U(n, n ; A)_{0}$, instead of by the commutator subgroups (which are always contained in the former), then we obtain in place of $K_{1}(A)$ and ${ }_{1} L_{1}(A)$ groups which (again in the notation of [Kar3]) are denoted by $K_{1}^{\text {top }}(A)$ and ${ }_{1} L_{1}^{\text {top }}(A)$. Théorème 2.3 of $\left[\right.$ Kar3] shows that ${ }_{1} L_{1}^{\text {top }}(A)$ is canonically isomorphic to $K_{1}^{\text {top }}(A) \oplus K_{1}^{\text {top }}(A)$,

**This is close to, but not exactly, the form of the definition given in [Ran1, §1.6] using "formations," but is an equivalent formulation obtained by modifying the definition of [what is now called] $L_{1}^{s}(A)$ in [Wall, §6]. The class of a unitary $u \in U(n, n ; A)$ corresponds to the class of the formation

$$
\left(A^{n} \oplus A^{n}, A^{n} \oplus 0, u\left(A^{n} \oplus 0\right)\right) .
$$


with the natural map $K_{1}^{\mathrm{top}}(A) \rightarrow{ }_{1} L_{1}^{\mathrm{top}}(A)$ corresponding to the diagonal embedding. This means that we have an exact sequence

$$
K_{1}(A) \rightarrow U^{\text {hyp }}(A) / U^{\text {hyp }}(A)_{0} \rightarrow K_{1}^{\text {top }}(A) \rightarrow 0 .
$$

So to prove the theorem we need to show two things: that $\sigma_{n}$ is in $U(n, n$; $A)_{0}$, and that the image of $G L(n, A)_{0}$ in $U(n, n ; A)_{0}$ generates the latter modulo the commutator subgroup.

For our purposes it is useful to make use of the Lie algebra

$$
\mathfrak{g}=\left\{\left(\begin{array}{cc}
a & b \\
c & -a^{*}
\end{array}\right): b=-b^{*}, c=-c^{*}\right\}
$$

of $U(n, n ; A)$, which can be identified with the tangent space to $U(n, n ; A)_{0}$ at the identity. This space has the property that if $X \in \mathfrak{g}$, then $e^{t X} \in$ $U(n, n ; A)_{0}$. Since we are in the complex case,

$$
\left(\begin{array}{cc}
0 & i \\
i & 0
\end{array}\right),\left(\begin{array}{cc}
i & 0 \\
0 & i
\end{array}\right) \in \mathfrak{g}
$$

and exponentiating, we find that

$$
\begin{aligned}
\exp \left(\frac{-\pi}{2}\left(\begin{array}{ll}
i & 0 \\
0 & i
\end{array}\right)\right) \exp \left(\frac{\pi}{2}\left(\begin{array}{ll}
0 & i \\
i & 0
\end{array}\right)\right) & =\left(\begin{array}{cc}
-i & 0 \\
0 & -i
\end{array}\right)\left(\begin{array}{ll}
0 & i \\
i & 0
\end{array}\right) \\
& =\left(\begin{array}{ll}
0 & 1 \\
1 & 0
\end{array}\right)=\sigma_{n}
\end{aligned}
$$

Thus $\sigma_{n} \in U(n, n ; A)_{0}$. To prove that the image of $G L(n, A)_{0}$ generates $U(n, n ; A)_{0}$ modulo the commutator subgroup, we apply the (Banach space version of the) Implicit Function Theorem to the map

$$
\psi: G L(n, A)_{0} \times U(n, n ; A)_{0} \times U(n, n ; A)_{0} \rightarrow U(n, n ; A)_{0}
$$

defined by

$$
\left(h, g_{1}, g_{2}\right) \mapsto\left(\begin{array}{cc}
h & 0 \\
0 & \left(h^{*}\right)^{-1}
\end{array}\right) g_{1} g_{2} g_{1}^{-1} g_{2}^{-1}
$$

If we take (for example)

$$
g_{1}=\left(\begin{array}{cc}
2 & 0 \\
0 & \frac{1}{2}
\end{array}\right), \quad g_{2}=\exp \left(\begin{array}{cc}
0 & b \\
c & 0
\end{array}\right)
$$


we obtain

$$
g_{1} g_{2} g_{1}^{-1} g_{2}^{-1}=\exp \left(\begin{array}{cc}
0 & 4 b \\
\frac{1}{4} c & 0
\end{array}\right) \exp \left(\begin{array}{cc}
0 & -b \\
-c & 0
\end{array}\right)
$$

and from this it is easy to see that the differential of $\psi$ at the point $\left(1, g_{1}, 1\right)$ is surjective. Thus the image of $\psi$ contains an open neighborhood of the identity in $U(n, n ; A)_{0}$, and thus the image of $G L(n, A)_{0}$ and the commutator subgroup of $U(n, n ; A)_{0}$ generate all of $U(n, n ; A)_{0}$. This completes the proof.

If we look carefully at the above proof, we see that the assumption that $A$ is a complex (and not just a real) $C^{*}$-algebra was only used twice: once to show that the $L$-groups are 2-periodic, and once to show that $\sigma_{n} \in$ $U(n, n ; A)_{0}$. If $A$ is a real $C^{*}$-algebra, Karoubi's [Kar3, Théorème 2.3] is still valid, and thus the same proof as for Theorem 1.8 yields the following (which seems not to be in the literature).

1.9. Theorem. If $A$ is a real $C^{*}$-algebra, there is a canonical surjection of $K_{1}^{\text {top }}(A)$ onto $L_{1}^{h}(A)$, with kernel of order at most 2 (generated by the class of $\left.\sigma_{n}\right)$.

1.10. Remark. Note that one cannot do any better than this, since $K_{1}^{\text {top }}(\mathbb{R})$ $\cong \mathbb{Z} / 2$ and $L_{\text {odd }}^{p}$ vanishes for any semisimple ring [Ran1, Proposition 1.2.3(iii)], in particular for a field, so that $L_{1}^{p}(\mathbb{R})=L_{1}^{h}(\mathbb{R})=0$.

Now the Rothenberg exact sequence quoted in Remark 1.7 continues to the left as

$$
\begin{aligned}
L_{2}^{h}(A) & \rightarrow L_{2}^{p}(A) \rightarrow \hat{H}^{0}\left(\mathbb{Z} / 2 ; \tilde{K}_{0}(A)\right) \\
& \rightarrow L_{1}^{h}(A) \rightarrow L_{1}^{p}(A) \rightarrow \hat{H}^{1}\left(\mathbb{Z} / 2 ; \tilde{K}_{0}(A)\right) \rightarrow L_{0}^{h}(A),
\end{aligned}
$$

and since the map $\hat{H}^{1}\left(\mathbb{Z} / 2 ; \tilde{K}_{0}(A)\right) \rightarrow L_{0}^{h}(A)$ is injective, the map $L_{1}^{h}(A) \rightarrow$ $L_{1}^{p}(A)$ is always surjective. In the complex case where the map $L_{2}^{h}(A) \rightarrow$ $L_{2}^{p}(A)$ can be identified with the map $L_{0}^{h}(A) \rightarrow L_{0}^{p}(A)$, it follows from Remark 1.7 that $L_{1}^{h}(A) \rightarrow L_{1}^{p}(A)$ is always an isomorphism. However in the real case, the map $L_{1}^{h}(A) \rightarrow L_{1}^{p}(A)$ need not be an isomorphism. For if

$$
A=\mathbb{R} \oplus \cdots \oplus \mathbb{R} \quad(n \text { summands }),
$$

$A$ is still semisimple, so $L_{1}^{p}(A)=0$. And $L_{2}^{p}(A)=0$ since any symplectic form over $A$ is a hyperbolic form on a projective module. So from the Rothenberg exact sequence,

$$
L_{1}^{h}(A) \cong \hat{H}^{2}\left(\mathbb{Z} / 2 ; \tilde{K}_{0}(A)\right) \cong(\mathbb{Z} / 2)^{n-1} .
$$


This is consistent with Theorem 1.9 , since $K_{1}^{\mathrm{top}}(A) \cong(\mathbb{Z} / 2)^{n}$.

One may note as well that the projective $L$-groups $L_{*}^{p}$ have the good categorical properties

$$
\begin{gathered}
L_{*}^{p}\left(A_{1} \times A_{2}\right) \cong L_{*}^{p}\left(A_{1}\right) \oplus L_{*}^{p}\left(A_{2}\right) \quad \text { (additivity) }, \\
L_{*}^{p}\left(M_{n}(A)\right) \cong L_{*}^{p}(A) \quad \text { (Morita invariance) }
\end{gathered}
$$

[Ran2, §22] in analogy with $K$-theory. The free $L$-groups $L_{*}^{h}$ don't have such good properties, because the reduced projective class group $\widetilde{K}_{0}$ doesn't have them.

If we are willing to invert 2, then the results of Karoubi in [Kar1] and [Kar3] yield the following in the real case.

1.11. Theorem (essentially due to Karoubi). If $A$ is a real $C^{*}$-algebra, then the groups $K_{\bullet}^{\text {top }}(A)\left[\frac{1}{2}\right], L_{\bullet}^{h}(A)\left[\frac{1}{2}\right]$, and $L_{\bullet}^{p}(A)\left[\frac{1}{2}\right]$ are canonically isomorphic, and periodic with period 4.

Proof. Because the groups $\hat{H}^{\bullet}(\mathbb{Z} / 2$; _ $)$ are always 2-torsion, the Rothenberg sequence shows that (for any ring) the natural map $L_{\bullet}^{h}(A)\left[\frac{1}{2}\right] \rightarrow$ $L_{\bullet}^{p}(A)\left[\frac{1}{2}\right]$ is an isomorphism. Also it is well-known that the 8-periodicity of $K_{\bullet}^{\text {top }}(A)$ becomes a 4 -periodicity after inverting 2 (see for example [Kar2, Theorem III.2.11]). Since Theorems 1.6 and 1.9 already handle the cases $\bullet \cong 0,1 \bmod 4$, we need only explain how to deal with the cases $\bullet \cong 2,3$ mod 4 . Here we need to apply Théorème 0.1 and Théorème 0.4 of [Kar1], for which parts of the proofs appear in [Kar3] and [Kar4]. As in the case above, [Kar3, Théorème 2.3] shows that there is a natural isomorphism from ${ }_{1} L_{\bullet}^{\text {top }}(A)$ to $K_{\bullet}^{\text {top }}(A) \oplus K_{\bullet}^{\text {top }}(A)$, and thus from ${ }_{1} L_{\bullet}^{\text {top }}(A) / K_{\bullet}^{\text {top }}(A)=$ ${ }_{1} W_{\bullet}^{\text {top }}(A)$ (Karoubi's notation) to $K_{\bullet}^{\text {top }}(A)$. Now after inverting $2,{ }_{1} W_{\bullet}^{\text {top }}(A)$ becomes what Karoubi calls ${ }_{1} W_{\bullet}(A)\left[\frac{1}{2}\right][\operatorname{Kar} 1$, Théorème 0.1 and Théorème 0.4]. Applying periodicity, this is ${ }_{-1} W_{\bullet-2}(A)\left[\frac{1}{2}\right]$, which in turn coincides with $L_{\bullet-2}^{h}(A,-1) \cong L_{\bullet}^{h}(A)\left[\frac{1}{2}\right]$. (Karoubi's $W$-group is the same as Witt group, and thus the same as the Wall group, in degree 0, and differs from the usual $L$-group in degree 1 by at most a $\mathbb{Z} / 2$ coming from the class of $\sigma$.

1.12. It is worth pointing out (this fact is well-known to specialists in $C^{*}$ algebras) that the complex group ring $\mathbb{C} \pi$ of any group $\pi$ has a unique maximal $C^{*}$-algebra-completion, denoted $C_{\max }^{*}(\pi)$. It has the universal property that any involution-preserving homomorphism from $\mathbb{C} \pi$ into the algebra of bounded operators on a Hilbert space must factor through the canonical inclusion $\mathbb{C} \pi \hookrightarrow C_{\max }^{*}(\pi)$. Thus the study of generalized signatures naturally leads to a study of $K_{0}\left(C_{\max }^{*}(\pi)\right)$. Since the algebra $C_{\max }^{*}(\pi)$ is fairly 
intangible, however, most analytic proofs of the Novikov conjecture make do with the more concrete algebra $C_{r}^{*}(\pi)$ defined above, which is necessarily a quotient of $C_{\max }^{*}(\pi)$. The canonical map $C_{\max }^{*}(\pi) \rightarrow C_{r}^{*}(\pi)$ is an isomorphism if and only if $\pi$ is amenable. More generally, the group $\pi$ is called $K$-amenable when this map induces an isomorphism on topological $K$-groups. This condition is now known to be satisfied for discrete subgroups of $S O(n, 1)$ and $S U(n, 1)$, as well as for amenable groups. However it fails when $\pi$ has Kazhdan's property $\mathrm{T}$, for example, if $\pi$ is a lattice subgroup in $S p(n, 1)(n \geq 1)$ or in a simple Lie group of $\mathbb{R}$-rank $\geq 2$.

For purposes of studying which closed manifolds admit Riemannian metrics of positive scalar curvature, I was forced in [Ros1] and [Ros3] to deal with $C^{*}$-algebra completions of the real group ring $\mathbb{R} \pi$ rather than of the complex group ring $\mathbb{C} \pi$. This does not make an essential difference in the theory: one still has a universal $C^{*}$-algebra completion $C_{\max }^{*}(\pi)$ and a reduced $C^{*}$-algebra $C_{r}^{*}(\pi)$ (the completion of $\mathbb{R} \pi$ for its action on $\ell^{2}(\pi)$ by left multiplication). When it is necessary to distinguish this from the complex $C^{*}$-algebra, one can write $C_{r, \mathbb{R}}^{*}(\pi)$.

It seems that to the extent that there is a good $C^{*}$-algebraic analogue of the Borel conjecture (which involves surjectivity of an assembly map and

not just injectivity), this analogue (of which the leading candidate is called the Baum-Connes conjecture) must involve $C_{r}^{*}(\pi)$ and not $C_{\max }^{*}(\pi)$; the $K$-theory of the latter is in many cases simply "too large". Of course, for $K$-amenable groups, the question of which $C^{*}$-algebra completion of $\mathbb{C} \pi$ to use makes no difference.

\section{$\S 2$. Identity of the assembly and index maps}

Now that we have seen how the $K$-theory of $C^{*}$-algebras is naturally related to higher signatures, we come to the issue of proofs of the Novikov conjecture itself. Analytic proofs for a group $\pi$ tend to be arguments for injectivity of a certain index map, usually denoted $\mu$ or $\beta$, which roughly speaking maps the $K U$ - or $K O$-homology of $B \pi$ to the $K$-groups of some $C^{*}$-algebra completion of the real or complex group ring.

To relate this to the surgery-theoretic assembly map as defined, say, in [Ran2] or in [Q2], we need first of all a version of Theorems 1.8 and 1.11 on the level of spectra and not just on the level of individual groups. While it is in some sense known to the experts that such a version should exist, it does not seem to be written down anywhere, so we provide some details. But even in the complex case, just as in Theorem 1.11, it is necessary to invert 2 . The reason is basically that $L$-spectra when localized at 2 are always Eilenberg-MacLane spectra [TaW, Theorem A], whereas the spectra for topological $K$-theory behave quite differently at the prime 2 . 
2.1. Theorem. Let $A$ be a $C^{*}$-algebra, either real or complex, and let $\mathbb{L}_{\bullet}(A)$ be the $L$-theory spectrum of $A$ defined as in [RanL] and [Ran2]. (The $p$ decoration would be more natural here, but it really doesn't matter since we need to invert 2 anyway. $\mathbb{L}^{p}$ and $\mathbb{L}^{h}$ become equivalent after inverting 2.) Let $\mathbb{K}_{\bullet}^{\text {top }}(A)$ denote the spectrum for topological $K$-theory of $A$, with homotopy groups $K_{\bullet}^{\mathrm{top}}(A)$. (This may be realized as an $\Omega$-spectrum with 0th space $K_{0}(A) \times B G L(A)$. In the complex case, each even-numbered space may be taken to be $K_{0}(A) \times B G L(A)$ and each odd-numbered space may be taken to be $G L(A)$. In the real case, every 8-th space may be taken to be $K_{0}(A) \times B G L(A)$. Each space in the spectrum has the homotopy type of a $C W$-complex, by [Pal].) We localize the spectra as in [Ad, §3.1].

Then there is a natural equivalence of spectra

$$
\mathbb{K}_{\bullet}^{\mathrm{top}}(A)\left[\frac{1}{2}\right] \rightarrow \mathbb{L}_{\bullet}(A)\left[\frac{1}{2}\right]
$$

which on homotopy groups induces the isomorphisms of Theorems 1.8 and 1.11 .

Proof. The proof that follows is rather nonconstructive. It would be desirable to have a more constructive argument based on directly constructing a map of (unlocalized) infinite loop spaces which induces an equivalence on homotopy groups after localization, but we have encountered various difficulties in doing this.

First we observe that the theorem is true for $A=\mathbb{R}$. To see this, note that $\mathbb{L} \bullet(\mathbb{Z})$ is a periodic delooping of the infinite loop space

$$
\mathbb{L}_{0}(\mathbb{Z}) \simeq \mathbb{Z} \times G / \text { Top }
$$

[Ran2, p. 136], which after inverting 2 becomes equivalent to the 0th space $\mathbb{Z} \times B O$ in the spectrum of real $K$-theory $\mathbb{K}_{\bullet}^{\text {top }}(\mathbb{R})$ [MaM, Theorem 4.28]. The equivalence

$$
\mathbb{Z} \times G / \operatorname{Top}\left[\frac{1}{2}\right] \simeq \mathbb{Z} \times B O\left[\frac{1}{2}\right]
$$

can be chosen to respect the natural $H$-space structures (coming on the left from the Cartesian product of a surgery problem with a manifold, and coming on the right from the tensor product of vector bundles) [MaM, Corollary 4.31]. On the other hand, the equivalence is also an infinite loop map [MaM, beginning of Ch. 6]. The equivalence

$$
\mathbb{L}_{0}(\mathbb{Z}) \simeq \mathbb{Z} \times G / \text { Top }
$$

also respects the infinite loop space structures. The spectrum $\mathbb{L}$ • $(\mathbb{Z})$ is a $\mathbb{L} \bullet(\mathbb{Z})$-module spectrum [Ran2, Appendix B], in essence via the tensor product of a quadratic form with a symmetric form, and the multiplication

$$
\mathbb{L}^{\bullet}(\mathbb{Z}) \wedge \mathbb{L}_{\bullet}(\mathbb{Z}) \rightarrow \mathbb{L}_{\bullet}(\mathbb{Z})
$$


obviously corresponds to the "Cartesian product" $H$-space structure on $\mathbb{Z} \times G / \operatorname{Top}\left[\frac{1}{2}\right]$. So putting everything together and taking the periodic deloopings, we obtain an equivalence of spectra

$$
\mathbb{L}_{\bullet}(\mathbb{Z})\left[\frac{1}{2}\right] \simeq \mathbb{K}_{\bullet}^{\mathrm{top}}(\mathbb{R})\left[\frac{1}{2}\right]
$$

Furthermore, the inclusion $\mathbb{Z} \hookrightarrow \mathbb{R}$ induces isomorphisms on $L$-groups after inverting 2 , and the symmetrization map $\mathbb{L} \bullet(\mathbb{Z}) \rightarrow \mathbb{L} \bullet(\mathbb{Z})$ respects $\mathbb{L} \bullet(\mathbb{Z})$ module structures and becomes an equivalence after inverting 2 , so that we obtain equivalences of ring spectra

$$
\mathbb{K}_{\bullet}^{\text {top }}(\mathbb{R})\left[\frac{1}{2}\right] \simeq \mathbb{L}^{\bullet}(\mathbb{Z})\left[\frac{1}{2}\right] \stackrel{\simeq}{\longrightarrow} \mathbb{L}^{\bullet}(\mathbb{R})\left[\frac{1}{2}\right]
$$

The fact this equivalence is compatible with products amounts to saying that it matches up the respective periodicity operators (which shift degree by 4$)$.

Now consider a general $C^{*}$-algebra $A$. Then $\mathbb{K}_{\bullet}^{\text {top }}(A)$ is naturally a module spectrum over $\mathbb{K}_{\bullet}^{\text {top }}(\mathbb{R})$, while $\mathbb{L}_{\bullet}(A)$ is naturally a module spectrum over $\mathbb{L}^{\bullet}(\mathbb{R})$, in each case via the tensor product. And we have seen that the equivalence

$$
\mathbb{K}_{\bullet}^{\text {top }}(\mathbb{R})\left[\frac{1}{2}\right] \simeq \mathbb{L}^{\bullet}(\mathbb{R})\left[\frac{1}{2}\right]
$$

is compatible with products. So because of Theorems 1.8 and 1.11, it's enough to know:

2.2. Theorem (Bousfield). Two $\mathbb{K}_{\bullet}^{\text {top }}(\mathbb{R})\left[\frac{1}{2}\right]$-module spectra with the same homotopy groups are homotopy-equivalent. (More precisely, any isomorphism of the homotopy groups of such spectra, compatible with the module action of the ring

$$
\pi_{\bullet}\left(\mathbb{K}_{\bullet}^{\mathrm{top}}(\mathbb{R})\left[\frac{1}{2}\right]\right)=K_{\bullet}^{\mathrm{top}}(\mathbb{R})\left[\frac{1}{2}\right] \cong \mathbb{Z}\left[\frac{1}{2}\right]\left[t, t^{-1}\right],
$$

where $t$ is in degree 4 , is realized by an equivalence of spectra.)

Proof. This follows immediately from the Universal Coefficient Theorem for $\mathbb{K}_{\bullet}^{\text {top }}(\mathbb{R})$-module spectra of Bousfield [Bou, Theorems 4.6 and 9.6].

We want to use Theorem 2.1 to relate the analytic literature on the Novikov conjecture to the topological literature. For this we need to recall the basic construction in the analytic literature, the Mishchenko/Kasparov/Baum-Connes index map.

$\ddagger$ I am indebted to Stephan Stolz for pointing out to me the relevance of Bousfield's work. 
2.3. Definition. Let $\pi$ be a group, and let $C_{r}^{*}(\pi)$ denote its real or complex reduced $C^{*}$-algebra. (The construction works equally well with real and complex coefficients.) The index map $\beta$ (also sometimes denoted $\mu$ ) from $K_{\bullet}(B \pi)$ (meaning $K U_{\bullet}(B \pi)$ in the complex case, $K O \cdot(B \pi)$ in the real case) to $K_{\bullet}^{\text {top }}\left(C_{r}^{*}(\pi)\right)$, first studied in this generality in [Kas2], is defined as follows. First define the universal bundle $\mathcal{V}$ over $B \pi$ with fibers which are finitely generated projective (right) $C_{r}^{*}(\pi)$-modules, by letting $\mathcal{V}=E \pi \times_{\pi}$ $C_{r}^{*}(\pi)$. This defines a class $[\mathcal{V}]$ in the Grothendieck group of such bundles, $K^{0}\left(B \pi ; C_{r}^{*}(\pi)\right)$, first studied for this purpose in [MS]. Then $\beta$ is basically the operation of "slant product" with this class. To make this rigorous, think of $K_{\bullet}(B \pi)$ as $\lim K_{\bullet}(X)$, where $X$ runs over the finite subcomplexes of $B \pi$ indexed by inclusion. Then $[\mathcal{V}]$ pulls back to a class $[\mathcal{V}]_{X}$ over any such $X$, which we can think of as belonging to

$$
K^{0}\left(X ; C_{r}^{*}(\pi)\right) \cong K_{0}\left(C(X) \otimes C_{r}^{*}(\pi)\right),
$$

with $C(X) \otimes C_{r}^{*}(\pi)$ here denoting the (completed) tensor product of $C^{*}$ algebras, in other words, $C\left(X, C_{r}^{*}(\pi)\right)$. (Restriction to a finite subcomplex makes $X$ compact, and thus makes $C(X)$ a commutative $C^{*}$-algebra with unit.) The "slant product" to be used here is the Kasparov intersection product of [Kas1]

$$
K_{\bullet}(X) \cong K_{\bullet}(C(X), \mathbb{C}) \stackrel{[\mathcal{V}]_{X} \otimes x}{\longrightarrow} K K_{\bullet}\left(\mathbb{C}, C_{r}^{*}(\pi)\right) \cong K_{\bullet}^{\mathrm{top}}\left(C_{r}^{*}(\pi)\right), \dagger \dagger
$$

for which convenient expository references are [Fac1] and [Bla, §18].łł

Calling $\beta$ the "index map" is justified by the following interpretation, which explains precisely how it arises in the analytic proofs. Namely, let $M$ be a closed manifold equipped with a map $f: M \rightarrow B \pi$, and suppose $D$ is an elliptic operator on $M$, for example the signature operator on an oriented manifold, or the Dirac operator on a spin manifold. The definition of the Kasparov groups is such that $D$ immediately defines a class $[D] \in K_{\bullet}(M)$, which we can push forward to a class $f_{*}([D]) \in K_{\bullet}(B \pi)$. In fact, by a fairly easy theorem of Conner-Floyd type, all classes in $K \bullet(B \pi)$ arise this way (a much more precise result along these lines has been given by Baum, and is announced in $[\mathrm{BD}]$, though the complete proof never appeared). Then $\beta\left(f_{*}([D])\right) \in K_{\bullet}^{\text {top }}\left(C_{r}^{*}(\pi)\right)$ is the index in the sense of Mishchenko-Fomenko [MF] of $D \mathcal{V}$, meaning $D$ with "twisted coefficients" in the flat bundle $\mathcal{V}$.

$\dagger \dagger$ In the real case, replace $\mathbb{C}$ everywhere by $\mathbb{R}$.

$\ddagger \ddagger$ Since we are not using quite the most general case of the Kasparov product, there are various ways of simplifying the definition of the pairing here, for example by using the Connes-Higson notion of "asymptotic morphism" in [CoH]. 
This follows immediately from the interpretation of the Kasparov product in terms of index theory, explained in [Bla, §24] or in [Ros4].

2.4. As we will see in the next section, there is another way of defining $\beta$, which comes from going up from $B \pi$ to its universal cover $E \pi$ and doing everything equivariantly with respect to $\pi$. From this point of view, $\beta$ is the map in equivariant Kasparov $K$-homology defined by the $\pi$-equivariant "collapse map" $E \pi \rightarrow *$ to a point, followed by the isomorphism $K_{\bullet}^{\pi}(*) \cong$ $K_{\bullet}^{\text {top }}\left(C_{\max }^{*}(\pi)\right)[\operatorname{Kas} 2, \S 4]$ and the map on topological $K$-groups induced by the projection $C_{\max }^{*}(\pi) \rightarrow C_{r}^{*}(\pi)$ mentioned above in 1.12. Here we note that $K_{\bullet}^{\pi}(E \pi) \cong K_{\bullet}(B \pi)$ since $\pi$ acts freely on $E \pi$, so that we indeed get a map $K_{\bullet}(B \pi) \rightarrow K_{\bullet}^{\text {top }}\left(C_{r}^{*}(\pi)\right)$. Chasing the definitions of the various Kasparov products involved shows that in this case we get back to the previous construction of $\beta$.

We can justify the name "index map" for $\beta$ from this point of view as well. If $D$ is an elliptic operator on a closed manifold $M$, defining a class $[D] \in K_{0}(M)$, then its analytic index (in the usual sense) is exactly the image of $[D]$ in $K_{0}(*) \cong \mathbb{Z}$ under the "collapse map" $M \rightarrow *$. If $D$ commutes with an action of a compact group $G$ on $M$, then $D$ defines an equivariant class in equivariant $K$-homology $K_{0}^{G}(M)$, and its equivariant index is the image of this class in $K_{0}^{G}(*) \cong R(G)$ under the (G-equivariant) "collapse map" $M \rightarrow *$. (See [Bla, §24] and [Ros4] for an explanation.) What we are doing here is exactly analogous, except that we are replacing $G$ by $\pi$ and doing $\pi$-equivariant index theory on the covering space of $M$ defined by a $\operatorname{map} M \rightarrow B \pi$.

2.5. Remark. The map which we have just called $\beta$ is closely related to a similar map, often denoted $\mu$, which Baum and Connes have conjectured to be an isomorphism (see $[\mathrm{BCH}]$ ). The relationship is the following. One can define a $\pi$-space $\underline{E} \pi$ (see $[\mathrm{BCH}]$ ) which is universal for proper actions of $\pi$, just as $E \pi$ is universal for free actions. (When $\pi$ is finite, $\underline{E} \pi$ is just the one-point $\pi$-space $*$, while $E \pi$ is of course infinite-dimensional.) Then the Baum-Connes map $\mu: K_{\bullet}^{\pi}(\underline{E} \pi) \rightarrow K_{\bullet}^{\text {top }}\left(C_{r}^{*}(\pi)\right)$ can be defined similarly, as the map in equivariant Kasparov $K$-homology defined by the $\pi$-equivariant "collapse map" $\underline{E} \pi \rightarrow *$, followed by the isomorphism $K_{\bullet}^{\pi}(*) \cong K_{\bullet}^{\mathrm{top}}\left(C_{\max }^{*}(\pi)\right)$ and the map on topological $K$-groups induced by the projection $C_{\max }^{*}(\pi) \rightarrow C_{r}^{*}(\pi)$ mentioned above in 1.12. Since $\underline{E} \pi=E \pi$ if $\pi$ is torsion-free, in this case $\mu$ reduces to $\beta$. For groups with torsion, $\underline{E} \pi \times E \pi$ is a free contractible $\pi$-space, thus another model for $E \pi$, and thus projection onto the second factor gives (up to equivariant homotopy) a $\pi$-map $E \pi \rightarrow \underline{E} \pi$ through which the collapse map $E \pi \rightarrow *$ factors, and in this way $\beta$ factors through $\mu$. 
2.6. Now we address the question of how one gets from information about $\beta$ or $\mu$ to information about the Novikov conjecture. If one is only interested in homotopy invariance of higher signatures, it is not necessary to invoke spectra, and two arguments are available, one due to Kasparov, described in $[$ Kas2, §9] and in [Kas5], and the other due to Kaminker-Miller [KM]. These show directly (without any reference to $L$-theory assembly) that if $D$ is the signature operator on an oriented closed manifold $M^{2 n}$ equipped with a map $f: M \rightarrow B \pi$, then $\beta\left(f_{*}([D])\right) \in K_{\bullet}^{\text {top }}\left(C_{r}^{*}(\pi)\right)$ is an oriented homotopy invariant. Thus if $\beta$ is rationally injective, $\mathrm{Ch} f_{*}([D])=2^{n} f_{*}([M] \cap \mathcal{L}(M)) \in$ $H_{\bullet}(B \pi ; \mathbb{Q})$ is an oriented homotopy invariant, $\mathcal{L}$ being the Atiyah-Singer renormalization of the total Hirzebruch $L$-class. This conclusion is obviously equivalent to the usual formulation of the Novikov conjecture.

However, it is more interesting to ask how integral statements about $\beta$ are related to integral versions of the Novikov conjecture. Here Theorem 2.1 turns out to be quite relevant.

2.7. Theorem. Let $A^{h}$ and $A^{p}$ denote the assembly maps in the sense of [Ran2] for (periodic) $L^{h}$ - and $L^{p}$-theory, respectively. Then for any connected $C W$-complex $X$, the following diagram commutes:

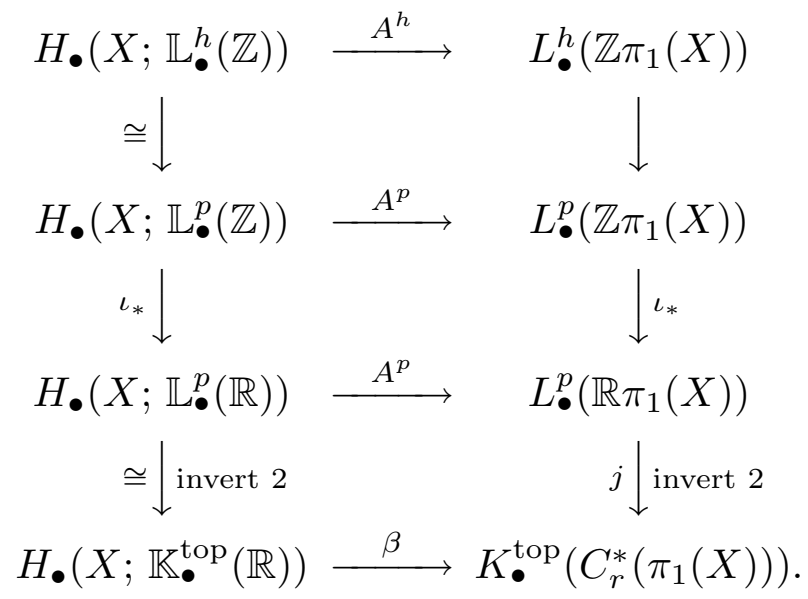

Here the maps $\iota_{*}$ are induced by the inclusions $\mathbb{Z} \hookrightarrow \mathbb{R}, \mathbb{Z} \pi_{1}(X) \hookrightarrow \mathbb{C} \pi_{1}(X)$, and the map $j$ is map on $L^{p}$-groups induced by the inclusion $\mathbb{C} \pi_{1}(X) \hookrightarrow$ $C_{r}^{*}\left(\pi_{1}(X)\right)$, followed by the isomorphism of Theorem 1.11. (One can also replace $\mathbb{R}$ by $\mathbb{C}$ in the diagram.)

Reduction of the problem. The commutativity of the upper two squares is obvious from the general machinery of [Ran2], so we need to prove commutativity of the lower square. Furthermore, the assembly maps are really the induced maps on homotopy groups of certain maps of spectra (which 
with minor abuse of notation we'll denote by the same letters), so we will do slightly better and show that that the diagram of spectra

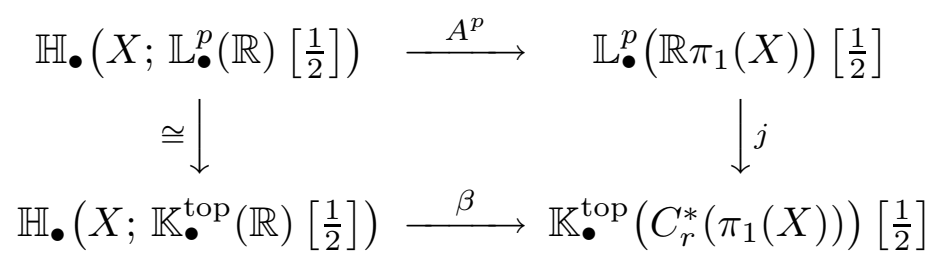

is homotopy-commutative. We sketch two different arguments for this.

First Proof. The first proof depends on the description of assembly for $L$ theory given in [Ran2, Appendix B], together with the homotopy-theoretic description of $\beta$ in [Ros3, Theorem 2.2] along the lines of the description of assembly in [Lod]. Let $f: X \rightarrow B \pi_{1}(X)$ be the classifying map for the universal cover of $X$. The references just cited give parallel descriptions of the maps $A^{p}$ and $\beta$ as composites

$$
\begin{aligned}
\mathbb{H}_{\bullet}\left(X ; \mathbb{L}_{\bullet}^{p}(\mathbb{R})\right)=X_{+} \wedge \mathbb{L}_{\bullet}^{p}(\mathbb{R}) \stackrel{f_{*} \wedge i d}{\longrightarrow} B \pi_{1}(X)+\wedge \mathbb{L}_{\bullet}^{p}(\mathbb{R}) \\
\stackrel{\sigma \wedge i d}{\longrightarrow} \mathbb{L}_{\bullet}^{p}\left(\mathbb{R} \pi_{1}(X)\right) \wedge \mathbb{L}_{\bullet}^{p}(\mathbb{R}) \stackrel{\mu}{\longrightarrow} \mathbb{L}_{\bullet}^{p}\left(\mathbb{R} \pi_{1}(X)\right), \\
\mathbb{H}_{\bullet}\left(X ; \mathbb{K}_{\bullet}^{\text {top }}(\mathbb{R})\right)=X_{+} \wedge \mathbb{K}_{\bullet}^{\text {top }}(\mathbb{R}) \stackrel{f_{*} \wedge i d}{\longrightarrow} B \pi_{1}(X)_{+} \wedge \mathbb{K}_{\bullet}^{\text {top }}(\mathbb{R}) \\
\stackrel{B \iota \wedge i d}{\longrightarrow} \mathbb{K}_{\bullet}^{\text {top }}\left(\mathbb{R} \pi_{1}(X)\right) \wedge \mathbb{K}_{\bullet}^{\text {top }}(\mathbb{R}) \stackrel{\mu}{\longrightarrow} \mathbb{K}_{\bullet}^{\text {top }}\left(C_{r}^{*}\left(\pi_{1}(X)\right)\right) .
\end{aligned}
$$

Here $\sigma$ is the "preassembly" map of [Ran2, Appendix B] (we are using the fact that symmetric and quadratic $L$-theory agree for algebras over a field of characteristic 0) and $\iota$ is the inclusion of $\pi_{1}(X)$ into $G L\left(C_{r}^{*}\left(\pi_{1}(X)\right)\right), B \iota$ is the induced map of classifying spaces (recall the "identity component" of $\mathbb{K}_{0}^{\text {top }}\left(C_{r}^{*}\left(\pi_{1}(X)\right)\right)$ is just $\left.B G L\left(C_{r}^{*}\left(\pi_{1}(X)\right)\right)\right)$, and $\mu$ in each case is an appropriate "multiplication" map. Now as shown in [Ran2, Appendix B], preassembly is compatible with Loday-type assembly; in other words, the following diagram commutes:

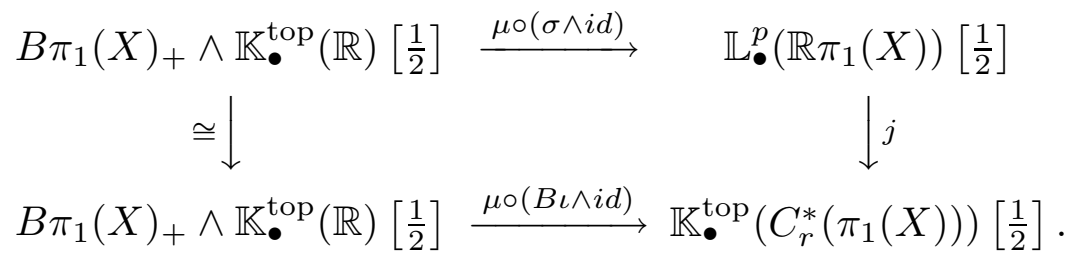

This clearly yields the desired result.

Second Proof. The second proof uses the abstract characterization of assembly maps found in [WW, §2]. (The idea that one could use the WeissWilliams results for this purpose I learned from reading [CarP].) The main result from $[\mathrm{WW}, \S 2]$ that we need is the following. 
2.8. Theorem [WW, §2]. Given a homotopy-invariant functor $F$ from spaces (without basepoint) to spectra, such that $F(\emptyset)$ is contractible, there is a unique strongly excisive homotopy-invariant functor (in other words, a generalized homology theory) $F^{\%}$ and a natural transformation $F^{\%} \rightarrow F$ (called assembly) which is the "best approximation to $F$ from the left by a homology theory," in the sense that given any strongly excisive homotopyinvariant functor $E$ with a natural transformation $\varphi: E \rightarrow F, E$ factors through $F$ \% . (Here "strongly excisive" means $F$ \% takes homotopy pushout squares of spaces to homotopy pushout squares of spectra, and preserves arbitrary coproducts, up to homotopy equivalence.)

2.9. Corollary (implicit in [WW, $\S 2]$ ). In the context of Theorem 2.8, there is a natural equivalence of functors

$$
F^{\%}(X) \simeq|X|_{+} \wedge F(*)
$$

and if $E$ is a strongly excisive homotopy-invariant functor with a natural transformation $\varphi: E \rightarrow F$, then $\varphi$ may be identified with the assembly for $F$ provided that

$$
\varphi(*): E(*) \rightarrow F(*)
$$

is an equivalence of spectra.

Proof. The first statement comes up in the proof of Theorem 2.8, and comes from the characterization of generalized homology theories in terms of spectra. Then if $E$ is a strongly excisive homotopy-invariant functor with a natural transformation $\varphi: E \rightarrow F$, we also have a natural equivalence

$$
E(X) \simeq|X|+\wedge E(*)
$$

and $\varphi$ factors through the assembly natural transformation $F^{\%} \rightarrow F$. This factorization $\varphi^{\%}$ gives an equivalence of spectra

$$
\varphi^{\%}(X): E(X) \simeq|X|_{+} \wedge E(*) \rightarrow|X|_{+} \wedge F(*) \simeq F^{\%}(X)
$$

for all $X$ provided that

$$
\varphi(*): E(*) \rightarrow F(*)
$$

is an equivalence of spectra.

In the context of [WW], "spaces" means CW-complexes, but as explained there, the theorem is valid on other categories of spaces, for example simplicial complexes. We want to apply the Weiss-Williams theorem to the functor

$$
F: X \mapsto \mathbb{K}_{\bullet}^{\text {top }}\left(C_{r}^{*}\left(\pi_{1}(X)\right)\right)
$$


but of course one encounters the difficulty that $\pi_{1}(X)$ depends on a choice of basepoint, and thus $X \mapsto \pi_{1}(X)$ is not well-defined on unbased spaces. To get around this, we use instead the $C^{*}$-algebra of the fundamental groupoid of a space, $\pi(X)$, which was also used in [RosW] for basically the same reason of functoriality. Here $\pi(X)$ is the topological groupoid with $X$ as its space of objects, whose morphisms from $x \in X$ to $y \in X$ are the homotopy classes $($ rel $\{0,1\}$ ) of paths $\gamma:[0,1] \rightarrow X$ with $\gamma(0)=x, \gamma(1)=y$. If $X$ is a connected space which is nice enough for covering space theory to apply, e.g., a CW-complex, then $\pi(X)$ can be identified with $\tilde{X} \times_{\Gamma} \tilde{X}$, where $\tilde{X} \rightarrow X$ is a universal covering space of $X$ and $\Gamma$ is the associated group of covering transformations, and thus carries a natural topology. If in addition $X$ is locally compact and carries a canonical measure class of full support (for instance, if $X$ is the geometric realization of a locally finite simplicial complex - we can take the class represented by Lebesgue measure on each simplex), then $\pi(X)$ is a locally compact groupoid admitting a Haar system (the measure class is needed to define the Haar system), and $C_{r}^{*}(\pi(X))$ is well-defined in the sense of [Ren]. More simply (and this is what we'll do here), if $X$ is a simplicial complex or simplicial set (and now one can dispense with the local finiteness and the measure), one can interpret $\pi(X)$ to mean the simplicial fundamental groupoid of $X$, which has as its set of objects the vertices of $X$ (with the discrete topology), and whose morphisms from a vertex $x$ to a vertex $y$ are the homotopy classes (rel $\{0,1\}$ ) of simplicial paths $\gamma$ from some subdivision of $[0,1]$ into $X$, with $\gamma(0)=x, \gamma(1)=y$. While this is not literally the same groupoid as before, it is equivalent to it, and has the advantages that $C_{r}^{*}(\pi(X))$ is always well-defined, and that a simplicial map gives rise to a $*$-homomorphism of the associated $C^{*}$-algebras. Furthermore, $C_{r}^{*}(\pi(X))$ is strongly Morita equivalent to the $\left(C^{*}\right.$-algebraic) direct sum of the $C_{r}^{*}\left(\pi_{1}\left(|X|, x_{j}\right)\right)$, where we choose one basepoint $x_{j}$ in each component of $X$. (This results from a trivial modification of [RosW, Theorem 2.5] to cover the disconnected case.) A simplicial map of polyhedra gives a map of the associated fundamental groupoids that only depends of the homotopy class of the map, so

$$
F: X \mapsto \mathbb{K}_{\bullet}^{\text {top }}\left(C_{r}^{*}(\pi(X))\right)
$$

is a homotopy functor on the category of simplicial sets. (In fact, though we don't need to know it, the homotopy type of the spectrum $\mathbb{K}_{\bullet}^{\text {top }}(A)$ depends only very weakly on the $C^{*}$-algebra $A$; as we mentioned once before, since $\mathbb{K}_{\bullet}^{\text {top }}(A)$ is a $\mathbb{K}_{\bullet}^{\text {top }}(\mathbb{R})$-module spectrum, its homotopy type as a spectrum, after localizing away from 2 , only depends on the topological $K$-groups of $A$ as groups. This follows immediately from Theorem 2.2.) As such, $F$ comes 
with an assembly natural transformation

$$
F^{\%}(X) \simeq|X|_{+} \wedge F(*)=|X|_{+} \wedge \mathbb{K}_{\bullet}^{\mathrm{top}}(\mathbb{R}) \rightarrow \mathbb{K}_{\bullet}^{\mathrm{top}}\left(C_{r}^{*}(\pi(X))\right)
$$

The universality statement in Theorem 2.8, together with Corollary 2.9, now proves that the assembly $F^{\%} \rightarrow F$ coincides with $\beta$; in fact, it gives an easy proof that the two definitions of $\beta$ in 2.3 and 2.4 coincide. $^{* * *}$ And similarly,

$$
G: X \mapsto \mathbb{L}_{\bullet}^{p}(\mathbb{R} \pi(X))
$$

is a homotopy functor on the category of simplicial sets, and $G^{\%}$ coincides with $A^{p}$. Since $j$ is a natural transformation $G\left[\frac{1}{2}\right] \rightarrow F\left[\frac{1}{2}\right]$, it yields a homotopy-commutative square

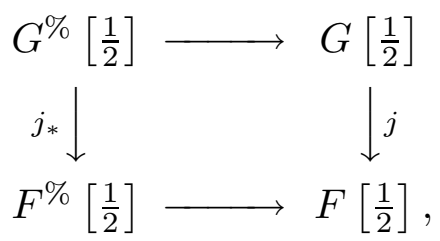

which is exactly what we needed.

This now yields an application to the integral Novikov conjecture.

2.10. Corollary. If, for some group $\pi$, the index map

$$
H_{\bullet}\left(B \pi ; \mathbb{K}_{\bullet}^{\text {top }}(\mathbb{R})\right) \stackrel{\beta}{\rightarrow} K_{\bullet}^{\text {top }}\left(C_{r}^{*}(\pi)\right)
$$

\footnotetext{
***In order to apply the argument, one needs to know that $\beta$ (using either the definition in 2.3 or the one in 2.4) may be viewed not just as a map of groups but also as a natural transformation of homotopy functors from spaces to spectra. We explain how to do this from the perspective of 2.3 ; the case of 2.4 is similar. Recall that we defined $K_{\bullet}(B \pi)$ as $\lim _{\bullet} K_{\bullet}(X)$, where $X$ runs over the finite subcomplexes of $B \pi$ indexed by inclusion, and $\vec{\beta}$ was defined using the Kasparov intersection product (over $C(X)$ ) with the class $[\mathcal{V}]_{X} \in K^{0}\left(X ; C_{r}^{*}(\pi)\right) \cong K_{0}\left(C(X) \otimes C_{r}^{*}(\pi)\right)$. Regard $K_{\bullet}(X)$ as the coefficient groups of a homology theory

$$
Y \mapsto K_{\bullet}(X \times Y)=K K_{\bullet}(C(X) \otimes C(Y), \mathbb{R})
$$

and $K_{\bullet}^{\text {top }}\left(C_{r}^{*}(\pi)\right)$ as the coefficient groups of the homology theory

$$
Y \mapsto K K_{\bullet}\left(C(Y), C_{r}^{*}(\pi)\right)
$$

Then the more general form of the Kasparov product (with "coefficients") gives a map of homology theories, and it's easy to check naturality.
} 
is injective (after inverting 2), then so is the $L$-theory assembly map

$$
H_{\bullet}\left(X ; \mathbb{L}_{\bullet}^{p}(\mathbb{R})\right) \stackrel{A^{p}}{\longrightarrow} L_{\bullet}^{p}\left(\mathbb{R} \pi_{1}(X)\right)
$$

(after inverting 2). Similarly, if $\beta\left[\frac{1}{2}\right]$ is split injective, then so is $A^{p}\left[\frac{1}{2}\right]$.

Proof. This is immediate from the commutative diagram in Theorem 2.7.

Of course, we can also work with complex instead of real $C^{*}$-algebras.

\section{$\S 3$. Bounded and controlled surgery}

In this final section, we will try to relate the topologists' notions of bounded and controlled surgery to the analytic work on the Novikov conjecture. While bounded and controlled surgery deal with non-compact surgery problems, with measurement of distances by means of reference maps to some metric space, they can be applied to problems about compact manifolds merely by passage to the universal cover. As such they are naturally related to the classical Novikov conjecture, as well of course to certain "coarse" analogues that may not be related to compact problems. The idea of passage from surgery on compact manifolds, related to the Novikov conjecture, to bounded or controlled surgery on the non-compact universal cover, makes an appearance in [Ran2, Appendix C], in [CarP], in [Gr], in [FW], in [Ran3], and in many other references which I will not attempt to enumerate here. Similarly, there is starting to be a considerable parallel literature on the analytic side, typified by [Roe1], [Roe2], and [HR2]. I will not attempt a "grand synthesis" of all of this work, but rather something much more modest. Namely, one sees in [CarP] and in [Ran2, Appendix C, $\S \mathrm{C} 4]$ the basic point of view that assembly maps can be viewed as "partial forget control" maps, from continuous control to bounded control. $\dagger \dagger \dagger$ The secret to splitting assembly maps, in other words to proving versions of the Novikov conjecture, thus seems to be to find a way to go back, from bounded control to continuous control. This in fact is the main theme in the analytic literature as well.

We begin with a review of some of the relevant algebraic and topological concepts.

$\dagger \dagger \dagger$ In fact, this point of view first appeared in [Q1], though the treatment there of what later came to be known as assembly is somewhat different, and harder to match up with what we are doing here.

To be honest, there are several competing notions of control that are relevant here, and the kind of "continuous control" used in $[\mathrm{CarP}]$ is different from what we call "continuous control" below, which is also known as "asymptotic control." Our approach to assembly here is closer to that in $[\mathrm{Car} 1, \S \mathrm{III}]$ than to that in [CarP]. However, the basic philosophy is pretty much the same. 
3.1. Definition. Let $(X, d)$ be a metric space and let $R$ be a ring. Let $\mathcal{C}_{X}(R)$ be the $X$-bounded projective $R$-module additive category of Pedersen and Weibel [PW]. This is defined as follows: an object in $\mathcal{C}_{X}(R)$ is a direct sum of finitely generated projective $R$-modules graded by $X$

$$
A=\bigoplus_{x \in X} A(x)
$$

such that for every subspace $K \subseteq X$ of finite diameter, only finitely many of the modules $A(x), x \in K$, are non-zero. A morphism $A \rightarrow B$ in $\mathcal{C}_{X}(R)$ is roughly speaking a matrix of finite bandwidth (called by some authors finite propagation distance), in other words, an $R$-module morphism defined by "matrix entries"

$$
f=\{f(y, x): A(x) \rightarrow B(y)\}
$$

such that there exists a number $b>0$ with $f(y, x)=0: A(x) \rightarrow B(y)$ for all $x, y \in X$ with $d(x, y)>b$. For $X$ compact, the "finite bandwidth" condition is always trivially satisfied, so $\mathcal{C}_{X}(R)$ is just equivalent to the category of finitely generated projective $R$-modules. If $R$ is a ring with involution $*$, then there is an induced involution on $\mathcal{C}_{X}(R)$ defined by applying * "pointwise" in $X$, and so the $L$-theory of $\mathcal{C}_{X}(R)$ is defined.

3.2. The Pedersen-Weibel category $\mathcal{C}_{X}(R)$ was originally introduced in [PW] for the purpose of giving a good realization of $K$-homology, since it turns out by the main theorem of $[\mathrm{PW}]$ that if $X=O(Y) \subseteq \mathbb{R}^{N+1}$ is the open cone on a compact subset $Y \subseteq S^{N}$, then

$$
K_{\bullet}\left(\mathcal{C}_{X}(R)\right) \cong H_{\bullet}^{l f}\left(O(Y) ; \mathbb{K}_{\bullet}(R)\right) \cong \tilde{H}_{\bullet-1}\left(Y ; \mathbb{K}_{\bullet}(R)\right) .
$$

Here $\mathbb{K}_{\bullet}(R)$ is the (nonconnective) algebraic $K$-theory spectrum of the ring $R$. When $R=\mathbb{R}$, the algebraic $K$-theory spectrum $\mathbb{K}_{\bullet}(\mathbb{R})$ maps to the topological $K$-theory spectrum $\mathbb{K}_{\bullet}^{\text {top }}(\mathbb{R})$, and so $K_{\bullet}\left(\mathcal{C}_{X}(R)\right)$ maps to the topological $K$-homology of $Y$ (with a degree shift). A direct construction of this map in terms of the Kasparov realization of topological $K$-homology was attempted in [Ros2, Theorem 3.4], but as John Roe has kindly pointed out to me, the proof given there is incorrect. (In the notation of the proof of [Ros2, Theorem 3.4], the error is that there is no reason why the action $\psi$ of $C(X)$ on the algebraic direct sum of the $A_{t \cdot x}$ should be a $*$-homomorphism with respect to the inner product $\langle\cdot, \cdot\rangle_{0}$.) However, we still ought to be able to match up "bounded topology" with the analytic literature based on $K K$-theory, and indeed this is done in [PRW], though only by nonconstructive methods and in the case where $X$ is the open cone on a compact polyhedron. Matching the "bounded topology" Novikov conjecture with the 
analytic literature on the Novikov conjecture involves the "simply connected assembly map" $A^{l f}$ of $\left[\right.$ Ran2, Appendix C] for the $L$-theory of $\mathcal{C} \tilde{X}_{\tilde{X}}(\mathbb{Z})$, where $\tilde{X}$ is the universal cover of a space $X$, and relating it by transfer to the usual assembly map

$$
A^{h}: H_{\bullet}\left(X ; \mathbb{L}_{\bullet}^{h}(\mathbb{Z})\right) \rightarrow L_{\bullet}^{h}\left(\mathbb{Z} \pi_{1}(X)\right) .
$$

However, we have to take the action of $\pi_{1}(X)$ into account and look at equivariant theories.

Recall that if a group $\pi$ acts on a (based) space $X$, the homotopy fixed set $X^{h \pi}$ is defined to be the set

$$
X^{h \pi}:=\operatorname{Map}\left(E \pi_{+}, X\right)^{\pi}
$$

of based equivariant maps from the universal $\pi$-space $E \pi_{+}$to $X$ (we've added a disjoint basepoint to $E \pi$ in order to stay in the based category). The obvious (based) map from the actual fixed set $X^{\pi}$ to the homotopy fixed set $X^{h \pi}$ may or may not be an equivalence.

We will need the observation that if $\tilde{X}$ is the universal covering of a connected compact polyhedron with fundamental group $\pi$ and if $R$ is a ring, then $\pi$ operates on the category $\mathcal{C}_{\tilde{X}}(R)$. If $R$ is a PID (so that all projective $R$-modules are free and $\left.\mathbb{L}_{\bullet}^{p}(R)=\mathbb{L}_{\bullet}^{h}(R):=\mathbb{L}_{\bullet}(R)\right)$, then in the definition of the category $\mathcal{C}_{\tilde{X}}(R)$ we may as well replace the word "projective" by the word "free," and the subcategory of invariants for this action may be identified with $\mathcal{C}_{X}^{\text {free }}(R \pi)$. This is defined the same way as $\mathcal{C}_{X}(R \pi)$, but again with the word "projective" replaced by the word "free," since $R \pi$ is exactly the free $R$-module with $\pi$ as basis, and since $R \pi$-module morphisms of finitely generated free $R \pi$-modules have finite bandwidth. The category $\mathcal{C}_{X}^{\text {free }}(R \pi)$ in turn is equivalent to the category of finitely generated free $R \pi$-modules, since $X$ is compact. Thus

$$
\mathbb{L}_{\bullet}\left(\mathcal{C}_{\tilde{X}}(R)\right)^{\pi} \simeq \mathbb{L}_{\bullet}^{h}(R \pi)
$$

(this observation was first made in $[\mathrm{CarP}]$ ), while

$$
\mathbb{H}\left(X ; \mathbb{L}_{\bullet}(R)\right) \simeq \mathbb{H}^{l f}\left(\tilde{X} ; \mathbb{L}_{\bullet}(R)\right)^{\pi} \simeq \mathbb{H}^{l f}\left(\tilde{X} ; \mathbb{L}_{\bullet}(R)\right)^{h \pi}
$$

if $\pi$ is torsion-free (this latter fact is proved in [Car1, Corollary II.5], [Car2, $\S \mathrm{II}]$ and in $[\mathrm{CarP}]$, in fact for general spectra in place of $\left.\mathbb{L}_{\bullet}(R)\right)$.

3.3. Theorem $[\mathrm{CarP}],[\mathrm{Ran} 3, \S 8]$. If $X$ is a connected compact polyhedron with torsion-free fundamental group $\pi$ and universal covering $\tilde{X}$, and $R$ is 
a PID (we are interested in the cases $\mathbb{Z}, \mathbb{R}$, and $\mathbb{C}$ ), then there is a natural homotopy-commutative diagram

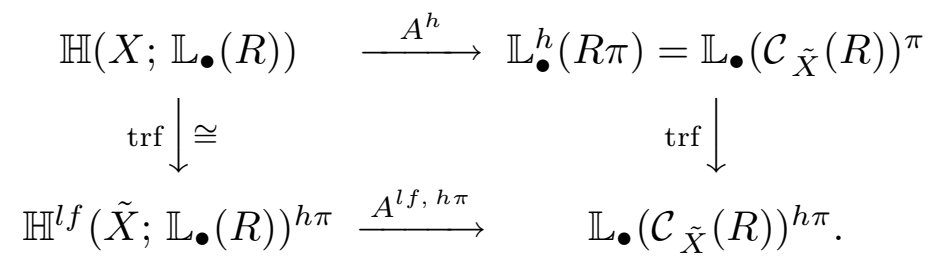

The vertical arrows may be identified with the natural maps from fixed sets to homotopy fixed sets.

In particular, if $\mathbb{H}^{l f}\left(\tilde{X} ; \mathbb{L}_{\bullet}(R)\right) \stackrel{A^{l f}}{\longrightarrow} \mathbb{L}_{\bullet}\left(\mathcal{C}_{\tilde{X}}(R)\right)$ is an equivalence, then (since this implies $A^{l f, h \pi}$ is also an equivalence) $A^{h}$ is split injective.

3.4. The rough idea of the methods of $[\mathrm{CarP}]$ (this is not exactly what they do, but only for technical reasons) is first to identify $H^{l f}\left(\tilde{X} ; \mathbb{L}_{\bullet}(R)\right)$ with the $L$-theory of a continuously controlled (and not just bounded) category of $R$-modules over $\tilde{X}$, and to identify the locally finite assembly map

$$
\mathbb{H}^{l f}\left(\tilde{X} ; \mathbb{L}_{\bullet}(R)\right) \stackrel{A^{l f}}{\longrightarrow} \mathbb{L}_{\bullet}\left(\mathcal{C}_{\tilde{X}}(R)\right)
$$

with a "partial forget control" map. Then if there is a model for $E \pi$ with a contractible metrizable compactification $Y$ on which $\pi$ acts, so that compact subsets of $E \pi$ become small when translated toward the boundary, one tries to use the geometry of the compactification to show that

$$
\mathbb{H}^{l f}\left(E \pi ; \mathbb{L}_{\bullet}(R)\right) \stackrel{A^{l f}}{\longrightarrow} \mathbb{L}_{\bullet}\left(\mathcal{C}_{E \pi}(R)\right)
$$

is a weak homotopy equivalence. The idea (which is also basic to [FW] and to many of the other references we have cited) is that "pushing to the boundary" converts bounded control to continuous control, and thus provides a splitting to the forgetful map. If one can show this, then one can apply Theorem 3.3 to finish the argument.

We will see that all of this has a counterpart in the analytic literature as well. One could trace this through almost all of the analytic papers on the Novikov conjecture, especially in [Mis3], [HsR], $[\mathrm{Gr}]$, and $[\mathrm{Hu}]$, but to the limit the discussion I will concentrate here on the programs of Kasparov and Roe as expressed, say, in [Kas4], [Kas5], [Roe2], and [HR2].

Now let's specialize to the case where the coefficient ring $R$ is $\mathbb{R}$ or $\mathbb{C}$. We'll write everything out for the case of $\mathbb{R}$, but $\mathbb{C}$ works exactly the same way. Following the principles which we have discussed earlier in this paper, 
the analytic counterpart to $\mathbb{L}_{\bullet}\left(\mathcal{C}_{\tilde{X}}(\mathbb{R})\right)$ should be the topological $K$-theory spectrum of a certain " $C^{*}$-completion" of the category $\mathcal{C}_{\tilde{X}}(\mathbb{R})$. There are a number of technical problems in defining this completion (which also came up in $[\operatorname{Ros} 2, \S 3])$, which are due to the fact that morphisms in the category $\mathcal{C}_{\tilde{X}}(\mathbb{R})$ are not necessarily bounded for any obvious norm, though they are "locally bounded" over compact subsets of $\tilde{X}$. To see exactly what is going on it is convenient first to simplify the category slightly by using the observation that $\tilde{X}$, being the universal cover of a compact metrizable space with fundamental group $\pi$, is "coarsely equivalent" to $|\pi|$, the group $\pi$ viewed as a metric space with regard to the word length metric $d$ defined by some set of generators. Thus the category $\mathcal{C}_{\tilde{X}}(\mathbb{R})$ is equivalent to the category $\mathcal{C}_{|\pi|}(\mathbb{R})$, for which the underlying metric space $|\pi|$ is countable (as a set). Then we may form the separable Hilbert space $\mathcal{H} \cong \ell^{2}(\pi) \otimes \ell^{2}$ (completed tensor product) which is the Hilbert space direct sum of one copy $\ell_{g}^{2}$ of $\ell^{2}$ (the standard infinite-dimensional separable Hilbert space) for each element $g$ of $\pi$. Each morphism in $\mathcal{C}_{|\pi|}(\mathbb{R})$ may be identified with an unbounded operator on $\mathcal{H}$, more specifically, by the operator defined by a matrix with rows and columns indexed by $\pi$, where the $(g, h)$ matrix entry is a finite-rank operator from $\ell_{h}^{2}$ to $\ell_{g}^{2}$, and where this entry vanishes as soon as $d(g, h)$ is sufficiently large (the "finite bandwidth" condition). The set of these operators is a $\mathbb{R}$-algebra $A(|\pi|)$ with an involution $*$, which has the convenient property of "stability," i.e., $M_{n}(A(|\pi|))$ is naturally isomorphic to $A(|\pi|)$ itself. However, $A(|\pi|)$ is a ring without unit, since the identity operator on $\ell_{g}^{2}$ is not of finite rank. For most purposes this is not a problem, since $A(|\pi|)$ has an "approximate identity" consisting of distinguished self-adjoint idempotents $p_{\alpha}$, where $\alpha$ ranges over all maps $\pi \rightarrow \mathbb{N}$, in the sense that $A(|\pi|)=\lim _{\alpha} p_{\alpha} A(|\pi|) p_{\alpha}$. (Cf. [HR1, Lemma 3.8].) The idempotent $p_{\alpha}$ is given by the diagonal matrix whose $(g, g)$-matrix entry is the orthogonal projection from $\ell_{g}^{2}$ onto the span of the first $\alpha(g)$ basis vectors. There is a naturally partial ordering on the maps $\alpha: \pi \rightarrow \mathbb{N}$, which corresponds to the usual partial order on idempotents as applied to the $p_{\alpha}$, coming from the usual order $\leq$ on $\mathbb{N}$. Note also that the objects of the category $\mathcal{C}_{|\pi|}(\mathbb{R})$ are in natural bijection with the $p_{\alpha}$ 's, so that for example an algebraic Poincaré complex in $\mathcal{C}_{|\pi|}(\mathbb{R})$ corresponds to an algebraic Poincaré complex of projective modules over some $p_{\alpha} A(|\pi|) p_{\alpha}$. Thus one can identify the $L$-theory spectrum of the category $\mathcal{C}_{\tilde{X}}(\mathbb{R})$ with the $L^{p}$-theory spectrum of the nonunital ring $A(|\pi|)$, by which we mean $\operatorname{hocolim}_{\alpha} \mathbb{L}^{p}\left(p_{\alpha} A(|\pi|) p_{\alpha}\right)$. (Here we are implicitly using the fact that $\mathbb{L}^{p}$, though not $\mathbb{L}^{h}$, is functorial for ring $*$-homomorphisms which are non-unital, but which map the unit of the first ring to a proper idempotent in the second.) 
Now let $A^{\text {bdd }}(|\pi|)$ be the subring of $A(|\pi|)$ consisting of matrices which act as bounded operators on $\mathcal{H}$. This is a $*$-closed algebra of Hilbert-space operators, so it has a $C^{*}$-algebra completion $C^{*}(|\pi|)$. It is trivial to see that this is the same as the algebra of the same name introduced in [Roe2] and [HR1, Definition 3.5]. Now we can give an analytic counterpart to Theorem 3.3 .

3.5. Theorem. In the situation of Theorem 3.3, the following diagram is homotopy-commutative. Here the map $i_{*}$ is induced by the inclusion $i$ : $A^{\text {bdd }}(|\pi|) \hookrightarrow A(|\pi|)$ and (to save space) we have omitted explicit mention of the fact that everything is to be localized away from 2 .

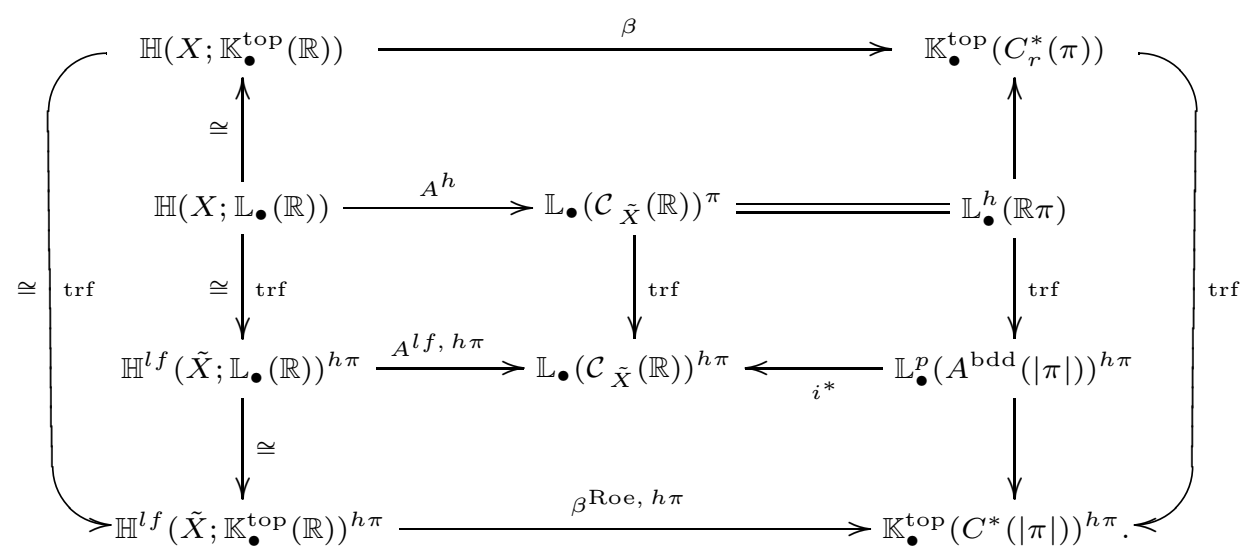

The horizontal map on the bottom is the analogue of $\beta$ in "coarse BaumConnes" theory, as explained in [HR2]. In fact, the outer square in this diagram is the "spacified" version of a diagram appearing at the end of $\S 6$ of [HR2].

Proof. Most of this follows immediately from Theorem 2.7 and Theorem 3.3. The fact that the middle and outer vertical arrows on the left are equivalences is, as we mentioned before, proved in [Car1, Corollary II.5], $[\mathrm{Car} 2, \S \mathrm{II}]$ and in $[\mathrm{CarP}]$. We only need to explain the arrows and the commutativity on the far right and very bottom of the diagram. The map $\mathbb{K}_{\bullet}^{\text {top }}\left(C_{r}^{*}(\pi)\right) \stackrel{\operatorname{trf}}{\longrightarrow} \mathbb{K}_{\bullet}^{\text {top }}\left(C^{*}(|\pi|)\right)^{h \pi}$, as explained at the end of $\S 6$ of [HR2], arises from the fact that $\pi$ acts naturally on $C^{*}(|\pi|)$, with fixed-point algebra Morita-equivalent (in the $C^{*}$-algebra sense) to $C_{r}^{*}(\pi)$. Thus the vertical arrow on the far right is induced by the inclusion $C^{*}(|\pi|)^{\pi} \hookrightarrow C^{*}(|\pi|)$. Similarly, $\pi$ acts naturally on $A^{\text {bdd }}(|\pi|)$, with fixed-point algebra (algebraically) Morita-equivalent to $\mathbb{R} \pi$, so one has a similar vertical arrow $\operatorname{trf}: \mathbb{L}_{\bullet}^{h}(\mathbb{R} \pi) \rightarrow$ $\mathbb{L}_{\bullet}^{p}\left(A^{\text {bdd }}(|\pi|)\right)^{h \pi}$ induced by the inclusion $A^{\text {bdd }}(|\pi|)^{\pi} \hookrightarrow A^{\text {bdd }}(|\pi|)$, and this arrow and trf : $\mathbb{K}_{\bullet}^{\text {top }}\left(C_{r}^{*}(\pi)\right) \rightarrow \mathbb{K}_{\bullet}^{\text {top }}\left(C^{*}(|\pi|)\right)$ fit together in a homotopycommutative square. The horizontal arrow at the bottom of the diagram 
is result of passing to homotopy fixed sets in the "spacified" version of the composite

$$
K_{\bullet}^{l f}(\tilde{X}) \rightarrow K_{\bullet}(\tilde{X}) \cong X_{\bullet}(|\pi|) \cong K_{\bullet}^{l f}(\underline{E} \pi) \stackrel{\mu}{\rightarrow} K_{\bullet}^{\text {top }}\left(C^{*}(|\pi|)\right)
$$

discussed in [HR2], where the commutativity of the outer square is also proved.

3.6. Corollary (basically discovered independently by several authors). If, in the situation of Theorem 3.3,

$$
\mathbb{H}^{l f}\left(\tilde{X} ; \mathbb{K}_{\bullet}^{\text {top }}(\mathbb{R})\right) \stackrel{\beta^{\text {Roe }}}{\longrightarrow} \mathbb{K}_{\bullet}^{\text {top }}\left(C^{*}(|\pi|)\right)
$$

is an equivalence, then (since this implies $\beta^{\mathrm{Roe}, h \pi}$ is also an equivalence) $A^{h}$ is split injective (after inverting 2).

Proof. Chase the diagram.

Now we can see in the analytic literature almost exact parallels to the topological theory. The coarse assembly map $\beta^{\text {Roe }}$ may again be regarded as a "partial forget control" map from continuously controlled to bounded topology. If there is a model for $E \pi$ with a contractible metrizable compactification $Y$ on which $\pi$ acts, so that compact subsets of $E \pi$ become small when translated toward the boundary, then "pushing to the boundary" converts bounded control to continuous control, and thus provides a splitting to the coarse assembly map. Corollary 3.6 then gives a form of the integral Novikov conjecture.

Furthermore, one can do away with the assumption that $\pi$ be torsion-free. When $\pi$ has torsion, the natural conjecture is that of Baum and Connes (see 2.5 above) with $\underline{E} \pi$ replacing $E \pi$. This has a counterpart for $L$-theory as well. Work in progress of Carlsson, Pedersen, and Roe (among others) gives an analogue of Corollary 3.6 in this context.

\section{REFERENCES}

[Ad] J. F. Adams, Infinite Loop Spaces, Annals of Math. Studies, vol. 90, Princeton Univ. Press, Princeton, 1978.

$[\mathrm{BCH}]$ P. Baum, A. Connes, and N. Higson, Classifying space for proper actions and $K$-theory of group $C^{*}$-algebras, $C^{*}$-algebras: A 50-Year Celebration (R. S. Doran, ed.), Contemp. Math., Amer. Math. Soc., Providence, RI, 1994 (to appear).

[BD] P. Baum and R. G. Douglas, K-homology and index theory, Operator Algebras and Applications (R. V. Kadison, ed.), Proc. Symp. Pure Math., vol. 38 (Part 1), Amer. Math. Soc., Providence, RI, 1982, pp. 117-173.

[Bla] B. Blackadar, K-Theory for Operator Algebras, Math. Sciences Research Inst. Publications, vol. 5, Springer-Verlag, New York, Berlin, Heidelberg, London, Paris, Tokyo, 1986. 
[Bou] A. K. Bousfield, A classification of K-local spectra, J. Pure Appl. Algebra 66 (1990), 121-163.

[Car1] G. Carlsson, Proper homotopy theory and transfers for infinite groups, Algebraic Topology and its Applications (G. Carlsson, R. Cohen, W.-C. Hsiang, and J. D. S. Jones, eds.), M. S. R. I. Publications, vol. 27, Springer-Verlag, New York, 1994, pp. 1-14.

[Car2] G. Carlsson, Bounded K-theory and the assembly map in algebraic K-theory, these proceedings.

[CarP] G. Carlsson and E. Pedersen, Controlled algebra and the Novikov conjectures for $K$ - and L-theory, Topology (to appear).

[CoH] A. Connes and N. Higson, Déformations, morphismes asymptotiques et K-théorie bivariante, C. R. Acad. Sci. Paris Sér. I 311 (1990), 101-106.

[Fac1] T. Fack, K-théorie bivariante de Kasparov, Séminaire Bourbaki, 1982-83, exposé no. 605, Astérisque 105-106 (1983), 149-166.

[Fac2] T. Fack, Sur la Conjecture de Novikov, Index theory of Elliptic Operators, Foliations, and Operator Algebras (J. Kaminker, K. C. Millet, and C. Schochet, eds.), Contemp. Math., vol. 70, Amer. Math. Soc., Providence, RI, 1988, pp. 43-102.

[FRR] S. Ferry, A. Ranicki, and J. Rosenberg, A history and survey of the Novikov conjecture, these proceedings.

[FW] S. Ferry and S. Weinberger, A coarse approach to the Novikov conjecture, these proceedings.

[GM] I. M. Gelfand and A. S. Mishchenko, Quadratic forms over commutative group rings, and K-theory, Funct. Anal. and its Appl. 3 (1969), 277-281.

[Gr] M. Gromov, Geometric reflections on the Novikov conjecture, these proceedings.

[HR1] N. Higson and J. Roe, A homotopy invariance theorem in coarse cohomology and $K$-theory, Trans. Amer. Math. Soc. (to appear).

[HR2] N. Higson and J. Roe, On the coarse Baum-Connes conjecture, these proceedings.

[HsR] W.-C. Hsiang and H. D. Rees, Mishchenko's work on Novikov's conjecture, Operator algebras and K-theory (San Francisco, 1981) (R. G Douglas and C. Schochet, eds.), Contemp. Math., vol. 10, Amer. Math. Soc., Providence, RI, 1982, pp. 77-98.

[Hu S. Hurder, Exotic index theory and the Novikov conjecture, these proceedings.

$[\mathrm{KM}]$ J. Kaminker and J. Miller, Homotopy invariance of the analytic index of signature operators over $C^{*}$-algebras, J. Operator Theory 14 (1985), 113-127.

[Kar1] M. Karoubi, Périodicité de la K-théorie hermitienne, Hermitian K-Theory and Geometric Applications (H. Bass, ed.), Lecture Notes in Math., vol. 343, SpringerVerlag, Berlin, Heidelberg, and New York, 1973, pp. 301-411.

[Kar2] M. Karoubi, K-Theory: An Introduction, Grundlehren der math. Wissenschaften, vol. 226, Springer-Verlag, Berlin, Heidelberg, New York, 1978.

[Kar3] M. Karoubi, Théorie de Quillen et homologie du groupe orthogonal, Ann. of Math. 112 (1980), 201-257.

[Kar4] M. Karoubi, Le théorème fondamental de la K-théorie hermitienne, Ann. of Math. 112 (1980), 259-282.

[Kas1] G. G. Kasparov, The operator $K$-functor and extensions of $C^{*}$-algebras, Izv. Akad. Nauk SSSR, Ser. Mat. 44 (1980), 571-636; English translation, Math. USSR-Izv. 16 (1981), 513-572.

[Kas2] G. G. Kasparov, K-theory, group $C^{*}$-algebras, and higher signatures: Conspectus, I, II, preprint, Inst. for Chemical Physics of the Soviet Acad. of Sci., Chernogolovka; reprinted with annotations, these proceedings. 
[Kas3] G. G. Kasparov, Operator K-theory and its applications: elliptic operators, group representations, higher signatures, $C^{*}$-extensions, Proc. International Congress of Mathematicians, Warsaw, 1983, vol. 2, Polish Scientific Publishers, Warsaw, 1984, pp. 987-1000.

[Kas4] G. G. Kasparov, Equivariant KK-theory and the Novikov conjecture, Invent. Math. 91 (1988), 147-201.

[Kas5] G. G. Kasparov, Novikov's conjecture on higher signatures: the operator K-theory approach, Representation Theory of Groups and Algebras (J. Adams, R. Herb, S. Kudla, J.-S. Li, R. Lipsman, and J. Rosenberg, eds.), Contemporary Math., vol. 145, Amer. Math. Soc., Providence, 1993, pp. 79-100.

[KS] G. G. Kasparov and G. Skandalis, Groups acting on buildings, operator K-theory, and Novikov's conjecture, $K$-Theory 4, Special issue in honor of A. Grothendieck, part 4 (1991), 303-337.

[Lod] J.-L. Loday, K-théorie algébrique et représentations de groupes, Ann. scient. Éc. Norm. Sup. 9 (1976), 309-377.

[MaM] I. Madsen and J. Milgram, The Classifying Spaces for Surgery and Cobordism of Manifolds, Ann. of Math. Studies, vol. 92, Princeton Univ. Press, Princeton, NJ, 1979.

[Mil] J. Miller, Signature operators and surgery groups over $C^{*}$-algebras, Preprint, 1992.

[Mis1] A. S. Mishchenko, Infinite dimensional representations of discrete groups and higher signatures, Math. U.S.S.R.-Izv. 8 (1974), 85-111.

[Mis2] A. S. Mishchenko, $C^{*}$-algebras and K-theory, Algebraic Topology, Aarhus 1978 (J. L. Dupont and I. Madsen, eds.), Lecture Notes in Math., vol. 763, SpringerVerlag, Berlin, Heidelberg, and New York, 1979, pp. 262-274.

[Mis3] A. S. Mishchenko, Controlled Fredholm representations, these proceedings.

[MF] A. S. Mishchenko and A. T. Fomenko, The index of elliptic operators over $C^{*}$-algebras, Izv. Akad. Nauk SSSR, Ser. Mat. 43 (1979), 831-859; English translation, Math. USSR-Izv. 15 (1980), 87-112.

[MS] A. S. Mishchenko and Yu. P. Soloviev, Infinite-dimensional representations of fundamental groups, and formulas of Hirzebruch type, Dokl. Akad. Nauk SSSR 234 (1977), 761-764; English translation, Soviet Math. Dokl. 18 (1977), 767-771.

[Nov] S. P. Novikov, Algebraic construction and properties of Hermitian analogs of $K$-theory over rings with involution from the viewpoint of the Hamiltonian formalism. Applications to differential topology and the theory of characteristic classes, Izv. Akad. Nauk SSSR, Ser. Mat. 34 (1970), 253-288, 475-500; English translation, Math. USSR-Izv. 4 (1970), 257-292, 479-505.

[Pal] R. S. Palais, Homotopy theory of infinite dimensional manifolds, Topology 5 (1966), 1-16.

[Ped] G. K. Pedersen, $C^{*}$-algebras and their Automorphism Groups, London Math. Soc. Monographs, vol. 14, Academic Press, London and New York, 1979.

[PRW] E. K. Pedersen, J. Roe, and S. Weinberger, On the homotopy invariance of the boundedly controlled signature of a manifold over an open cone, these proceedings.

[PW] E. K. Pedersen and C. Weibel, K-theory homology of spaces, Algebraic Topology, Proc., 1986 (G. Carlsson R. L. Cohen, H. R. Miller, and D. C. Ravenel, eds.), Lecture Notes in Math., vol. 1370, Springer-Verlag, Berlin, Heidelberg, and New York, 1989, pp. 346-361.

[Q1] F. Quinn, Ends of maps, II, Invent. Math. 68 (1982), 353-424. 
[Q2] F. Quinn, Assembly maps in bordism-type theories, these proceedings.

[Ran1] A. Ranicki, Exact Sequences in the Algebraic Theory of Surgery, Princeton Math. Notes, vol. 26, Princeton Univ. Press, Princeton, 1981.

[Ran2] A. Ranicki, Algebraic L-Theory and Topological Manifolds, Cambridge Tracts in Math., vol. 102, Cambridge Univ. Press, Cambridge, 1992.

[Ran3] A. Ranicki, On the Novikov conjecture, these proceedings.

[RanL] A. Ranicki and N. Levitt, Intrinsic transversality structures, Pacific J. Math. 129 (1987), 85-144.

[Ren] J. Renault, A groupoid approach to $C^{*}$-algebras, Lecture Notes in Math., vol. 793, Springer-Verlag, Berlin, Heidelberg, and New York, 1980.

[Roe1] J. Roe, Hyperbolic metric spaces and the exotic cohomology Novikov conjecture, K-Theory 4 (1990), 501-512; Erratum, ibid. 5 (1991), 189.

[Roe2] J. Roe, Coarse cohomology and index theory on complete Riemannian manifolds, Mem. Amer. Math. Soc. no. 497 (1993).

[Ros1] J. Rosenberg, $C^{*}$-algebras, positive scalar curvature, and the Novikov conjecture, III, Topology 25 (1986), 319-336.

[Ros2] J. Rosenberg, $K$ and KK: topology and operator algebras, Operator Theory / Operator Algebras and Applications (W. B. Arveson and R. G. Douglas, eds.), Proc. Symp. Pure Math., vol. 51 (Part 1), Amer. Math. Soc., Providence, RI, 1990, pp. $445-480$.

[Ros3] J. Rosenberg, The KO-assembly map and positive scalar curvature, Algebraic Topology, Poznań 1989 (S. Jackowski, B. Oliver, and K. Pawałowski, eds.), Lecture Notes in Math., vol. 1474, Springer-Verlag, Berlin, Heidelberg, and New York, 1991, pp. 170-182.

[Ros4] J. Rosenberg, Review of Elements of $K K$-theory by K. K. Jensen and $K$. Thomsen, Bull. Amer. Math. Soc. 28 (1993), 342-347.

[RosW] J. Rosenberg and S. Weinberger, An equivariant Novikov conjecture, with an appendix by J. P. May, $K$-Theory 4, Special issue in honor of A. Grothendieck, part 1 (1990), 29-53.

[TaW] L. Taylor and B. Williams, Surgery spaces: formulae and structure, Algebraic topology, Waterloo, 1978, Lecture Notes in Math., vol. 741, Springer-Verlag, Berlin, Heidelberg, New York, 1979, pp. 170-195.

[Wall] C. T. C. Wall, Surgery on Compact Manifolds, London Math. Soc. Monographs, vol. 1, Academic Press, London and New York, 1970.

[WW] M. S. Weiss and B. Williams, Automorphisms of manifolds and algebraic K-theory: Finale, preprint, Univ. of Notre Dame.

Department of Mathematics, University of Maryland, College Park, MD 20742, U.S.A.

E-mail: jmr@math.umd.edu 